\title{
Fredholm determinant representation of the Painlevé II $\tau$-function
}

\author{
Harini Desiraju* \\ Scuola Internazionale Superiore di Studi Avanzati, \\ Via Bonomea, 265, 34136 Trieste, Italy.
}

\begin{abstract}
We formulate the generic $\tau$-function of the Painleve II equation as a Fredholm determinant of an integrable (Its-Izergin-Korepin-Slavnov) operator. The $\tau$-function depends on the isomonodromic time $t$ and two Stokes' parameters, and the vanishing locus of the $\tau$-function, called the Malgrange divisor is determined by the zeros of the Fredholm determinant.
\end{abstract}

\section{Contents}

1 Introduction

2 Setup 5

2.1 Parametrices ............................... 8

2.1 .1 Model problem ..................... 8

$2.1 .2 \quad$ Local parametrices . . . . . . . . . . . . . . . . 10

3 Reduction to a RHP along the imaginary axis 11

4 Integrable kernel and Fredholm determinant 17

4.1 LU decomposition . . . . . . . . . . . . . . . . . . . . . . 18

4.2 Integrable kernel . . . . . . . . . . . . . . . . . . . . . . . . . 19

4.3 Malgrange forms . . . . . . . . . . . . . . . . . . . . . . . 21

5 Proof of theorem 1

*harini.desiraju@sissa.it 


\section{Introduction}

Painlevé equations describe isomonodromic deformations of certain meromorphic linear ordinary differential equations. In the theory of isomonodromic deformations, the JimboMiwa-Ueno $\tau$-funciton is defind in terms of a closed 1 -form, $\omega_{J M U}[29$ by the formula

$$
\delta \log \tau_{J M U}:=\omega_{J M U}
$$

where $\delta$ denotes the total differential with respect to the 'deformation' parameters. In this paper, we study the isomonodromic $\tau$-function of the second order scalar nonlinear ordinary differential equation (ODE) in the complex domain of the form

$$
\frac{d^{2}}{d x^{2}} u(x)=x u(x)-2 u(x)^{3}, \quad x \in \mathbb{C},
$$

called the homogenous Painlevé II equation. It arises as a consistency (zero-curvature) condition for the following set of linear ODEs for the $2 \times 2$ complex valued matrix $\Phi(\lambda, x)$

$$
\begin{gathered}
\frac{d \Phi}{d \lambda}=\left[-i\left(4 \lambda^{2}+x+2 u^{2}\right) \sigma_{3}+4 \lambda u \sigma_{1}-2 v \sigma_{2}\right] \Phi, \\
\frac{d \Phi}{d x}=\left[-i \lambda \sigma_{3}+u \sigma_{1}\right] \Phi,
\end{gathered}
$$

where $v(x)=u_{x}$, and the Pauli matrices

$$
\sigma_{1}=\left(\begin{array}{ll}
0 & 1 \\
1 & 0
\end{array}\right), \sigma_{2}=\left(\begin{array}{cc}
0 & -i \\
i & 0
\end{array}\right), \sigma_{3}=\left(\begin{array}{cc}
1 & 0 \\
0 & -1
\end{array}\right) .
$$

The set of ODEs (1.2) are called the Flaschka-Newell (FN) Lax pair 18. The ODE 1.2 has an irregular singularity at $\lambda=\infty$ of Poincaré rank 3 which exhibits Stokes' phenomenon on six rays on the complex plane. Such solution has jumps on the Stokes' rays, specified by Stokes' matrices.

The generalized mondromy data described by Stokes' matrices are encoded in the jump matrix $G(\lambda, x) \in G L(2, \mathbb{C}), \lambda \in \Sigma$ on the contour $\Sigma \in \mathbb{C}$. The inverse problem consists of reconstructing the function

$$
\Psi(\lambda, x)=\Phi(\lambda, x) e^{-\frac{4}{3} \lambda^{3}-x \lambda}
$$

from the generalized monodromy data. This is achieved by solving the following RiemannHilbert problem (RHP) (see section 2)

$$
\begin{gathered}
\Psi_{+}(\lambda, x)=\Psi_{-}(\lambda, x) G(\lambda, x), \quad \lambda \in \Sigma \\
\Psi(\lambda, x)=\mathbb{1}+\mathcal{O}\left(\frac{1}{\lambda}\right), \text { as } \lambda \rightarrow \infty,
\end{gathered}
$$

where $\Psi_{ \pm}$indicate the boundary value of $\Psi$ from the left side and the right side respectively of the oriented contour $\Sigma$. With this data, the Malgrange form is defined as follows.

Definition 1. The Malgrange form associated with the RHP 1.5 is defined as [33]

$$
\omega_{\Sigma}=\int_{\Sigma} \frac{d \lambda}{2 \pi i} \operatorname{Tr}\left[\Psi_{-}^{-1} \frac{\partial \Psi_{-}}{\partial \lambda} \delta G G^{-1}\right]
$$

where $\delta$ denotes the total differential with respect to the isomonodromic parameter $x$. 
Since $\omega_{\Sigma}$, is a close one form in the space of isomonodromic parameters, one can define (locally) the corresponding $\tau_{\Sigma}$ function as

$$
\delta \log \tau_{\Sigma}=\omega_{\Sigma} .
$$

The Malgrange form is a logarithmic form in the sense that it has only simple poles with integer residues. The locus in the parameter space where the RHP problem (1.5) becomes unsolvable is called the Malgrange divisor because (in the language of algebraic geometry) it can be described locally as the zero level set of a local analytic function.

The general gist is that this local expression can be represented (in abstract terms) as a Fredholm determinant (see for example [36]). A concrete realization of this local function (a $\tau$-function) as a Fredholm determinant (possibly globally defined on an open dense set of parameters) is of practical interest since it potentially allows for numerical investigation of the Malgrange divisor.

This paper treads this line of approach by providing a concrete representation for the $\tau$-function of Painlevé II in terms of a Fredholm determinant expressed via explicit (albeit complicated) kernel. According with this general framework, the zeros of the $\tau$-function indicate the points where the RHP (i.e, the inverse monodromy problem) is not solvable.

It is well known that certain special solutions of Painlevé equations have a Fredholm determinant representation $[6,7,28,39,41,42]$. The recent works of Lisovyy, Cafasso, Gavrylenko [12, 23] provide a method to formulate the isomonodromic $\tau$-functions of general solutions of PIII, PV, PVI as Fredholm determinants. There are two key aspects to their construction. One is the property that the RHPs of these Painleve equations can be reduced on to a RHP on the unit circle. The second feature is that the jump on the unit circle enables the formulation of the $\tau$-function as "Widom constant". An important feature of their construction is that the local parametrices of the RHP of the Painlevé equations are described by known special functions which in turn act as 'building blocks' of the $\tau$-function. For example, the local parametrices of the Painlevé VI RHP are given by hypergeometric functions and the $\tau$-function is expressed as a Fredholm determinant of hypergeometric kernel.

A natural question then is whether the $\tau$-functions of Painlevé I, II, IV admit a Fredholm determinant representation. In a first step to answer this question in the case of Painleve II, the present author recently showed that the RHP corresponding to the special 1-parameter (Ablowitz-Segur) family of solutions to the Painleve II equation [38] can be recast as a RHP on the imaginary axis as opposed to the unit circle in [12], hinting at a similar structure for the general RHP of Painlevé II. As a consequence, the corresponding $\tau$-function (which is known to be the determinant of the Airy kernel [39]), can be formulated as a Widom constant.

In the case of the RHP of Painlevé II, it is known that the local parametrices are described by the parabolic cylinder functions $D_{\nu}(z)$ which we recall in Section 2, We then reduce the RHP to a RHP with a discontinuity on the imaginary axis in Section 3. However, we will see that the jump on the imaginary axis does not admit Birkhoff factorization and hence the technique to construct Fredholm determinants in [17] is not applicable to our case. Instead, we use a variation of the formalism in [2] namely, a lower, diagonal, upper triangular (LDU) factorization of the jump matrix to construct the $\tau$-function as a Fredholm determinant of an integrable (IIKS) 14, 28 operator with the parabolic cylinder functions acting as the 'building blocks'. In order to formulate our result, let

$$
t=(-x)^{3 / 2} .
$$


We encode the Stokes parameters $s_{1}$ and $s_{3}$ that define the Painlevé II RHP, (see 2.26), 2.30 below) by :

$$
\nu=-\frac{1}{2 \pi i} \log \left(1-s_{1} s_{3}\right), \quad h=-\frac{\sqrt{2 \pi}}{\Gamma(-\nu) s_{3}} e^{i \pi \nu},
$$

with $s_{1} s_{3} \neq 0$, which corresponds to non-integer values of $\nu$.

Theorem 1. The $\tau$-function of Painlevé II equation can be expressed in terms of a Fredholm determinant of an integrable operator $\widetilde{\mathcal{K}}$ as follows

$$
\partial_{t} \log \tau_{P I I}=\partial_{t} \log \operatorname{det}\left[\mathbb{1}_{L^{2}(i \mathbb{R})}-\widetilde{\mathcal{K}}\right]-\left[\frac{4 i \nu}{3}+\frac{2 \nu^{2}}{t}\right]+\mathcal{F}(t, \nu, h),
$$

where $\mathcal{F}(t, \nu, h)$ is a regular function of $t, h$ and $\nu$ defined in 5.3$)$. The kernel of $\widetilde{\mathcal{K}}$ takes the form

$$
\widetilde{K}(z, w)=\frac{\mathcal{C}(z, t)}{\mathcal{A}(z, t)} \varphi_{-}^{2}(w) \int_{i \mathbb{R}-\epsilon} \frac{d \widetilde{w}}{2 \pi i} \frac{\varphi_{-}^{-2}(\widetilde{w})}{(z-\widetilde{w})(\widetilde{w}-w)} \mathcal{A}(\widetilde{w}, t) \mathcal{B}(\widetilde{w}, t)
$$

with

$$
\begin{gathered}
\mathcal{A}(z, t)=\zeta^{\nu} \xi^{\nu} e^{\frac{2 i}{3} t}\left(e^{-\pi i \nu} D_{-\nu}(i \zeta) D_{-\nu}(i \xi)+\nu^{2} h^{-4} e^{2 \pi i \nu} D_{\nu-1}(\zeta) D_{\nu-1}(\xi)\right) \\
\mathcal{B}(z, t)=\left(\frac{z+1 / 2}{z-1 / 2}\right)^{2 \nu} \zeta^{\nu} \xi^{-\nu}\left(i h^{2} e^{-i \pi \nu} D_{-\nu}(i \zeta) D_{-\nu-1}(i \xi)+\nu h^{-2} e^{2 \pi i \nu} D_{\nu-1}(\zeta) D_{\nu}(\xi)\right)
\end{gathered}
$$

where $D_{\nu}$ is the parabolic cylinder function, $\zeta=\zeta(z, t), \xi=\xi(z, t)$ are given by

$$
\zeta \equiv \zeta(z, t)=2 t^{1 / 2} \sqrt{-\frac{4 i}{3} z^{3}+i z-\frac{i}{3}} ; \quad \xi=\zeta(-z, t)
$$

and

$$
\mathcal{C}(z, t)=\mathcal{B}(-z, t) ; \quad \varphi_{-}(w, t)=\int_{i \mathbb{R}-\epsilon} \frac{d w^{\prime}}{2 \pi i} \frac{\log \mathcal{A}\left(w^{\prime}, t\right)}{w-w^{\prime}}
$$

Some comments are in order.

1. The $\tau$-function 11.8 is defined on $\mathbb{C} \backslash\{0\}$ and is analytic in $t$. Refer to Remark 11 for the details.

2. The important point of theorem 1 is that the zeros of $\tau_{P I I}$, called the Malgrange divisor are determined solely by the zero locus of the Fredholm determinant. The Malgrange divisor is in one to one correspondence with the poles of the Painlevé II transcendent.

3. The Malgrange divisor could be calculated numerically by computing the Fredholm determinant in (1.8) employing the algorithm developed in (5).

4. The limit from the general $\tau$-function of Painlevé II in $(1.8)$ to the $\tau$-function of the Ablowitz-Segur family of solutions (determinant of the Airy kernel) in [17] is singular because $s_{3} s_{1}=1$ ( $\nu$ in 1.7 goes to infinity). 
Theorem 1 is the first step in deriving the Fourier series representation of Painlevé II tau-function obtained in [27]. Series representation of the $\tau$-function (1.8) can be obtained from the minor expansion of the Fredholm determinant on an appropriate basis. A similar computation for the case of the Ablowitz-Segur solutions of Painlevé II is worked out in [17]. Furthermore, we expect that the methods developed in this manuscript can be applied to some solutions of Painlevé I and IV equations. Finally, the study of the inhomogenous Painlevé II equation ([19], Ch.x5)

$$
u_{x x}=x u-2 u^{3}+\alpha
$$

requires a significant extension of the techiniques developed for the homogenous Painlevé II equation (1.1). Indeed the parameter $\alpha$ which we set to zero in (1.1) induces a monodromy at the origin and the ideas developed in this manuscript need a non-trivial generalization. The $\tau$-function of the general solution of $(1.13)$ has been expressed as a ratio of Hankel determinants in [30], [31]. Finally, we remark that the $\tau$-functions of rational solutions of Painlevé equations have an interpretation not as Fredholm determinants, but as determinants of some special polynomials [13].

\section{Acknowledgements.}

Thanks are due to Alexander Its, Marco Bertola, Oleg Lisovyy, Pasha Gavrylenko, and Tamara Grava for illuminating discussions and valuable suggestions. Part of the work was completed during visits to the Department of Mathematics and Statistics at Concordia University, and Department of Mathematical sciences at IUPUI, which were supported by H2020-MSCA-RISE-2017 PROJECT No. 778010 IPADEGAN.

\section{Setup}

We recall the RHP associated to the Flashka-Newell Lax pair [1.2) from [19]. The matrix $\Psi(\lambda, x) \in G L(2, \mathbb{C})$ satisfies the following RHP on the contour in fig. 1 .

\section{Riemann-Hilbert problem 1.}

- $\Psi(\lambda, x)$ is piecewise analytic for $\lambda \in \mathbb{C} \backslash \cup_{k=1}^{6} \gamma_{k}$,

$$
\gamma_{k}=\left\{\lambda \in \mathbb{C}: \arg \lambda=\frac{\pi}{6}+\frac{\pi}{3}(k-1)\right\}, k=1, \ldots, 6 .
$$

We define $\Psi(\lambda, x) \equiv \Psi_{k}$ in the respective sector $\Omega_{k}$ defined by

$$
\Omega_{k}=\left\{\lambda \in \mathbb{C}: \frac{\pi}{6}(2 k-3)<\arg \lambda<\frac{\pi}{6}(2 k-1)\right\}, k=1, \ldots, 6 .
$$

- For $\lambda \in \gamma_{k}$, the following boundary condition is satisfied.

$$
\Psi_{k+1}=\Psi_{k} S_{k},
$$

where the Stokes' matrices $S_{k}$ are alternatively lower or upper triangular

$$
S_{k}=\left(\begin{array}{cc}
1 & 0 \\
s_{k} e^{2 i \theta(\lambda, x)} & 1
\end{array}\right) \text { for } k \equiv 1 \bmod 2, \quad S_{k}=\left(\begin{array}{cc}
1 & s_{k} e^{-2 i \theta(\lambda, x)} \\
0 & 1
\end{array}\right) \text { for } k \equiv 0 \bmod 2,
$$

and the exponent $\theta(\lambda, x)=\frac{4}{3} \lambda^{3}+x \lambda$. The Stokes' parameters $s_{k}$ are constants satisfying the constraint

$$
s_{k+3}=-s_{k}, s_{1}-s_{2}+s_{3}+s_{1} s_{2} s_{3}=0 .
$$


- In the asymptotic limit of $\lambda$,

$$
\lim _{\lambda \rightarrow \infty} \Psi(\lambda, x)=\mathbb{1}+\mathcal{O}\left(\lambda^{-1}\right) .
$$

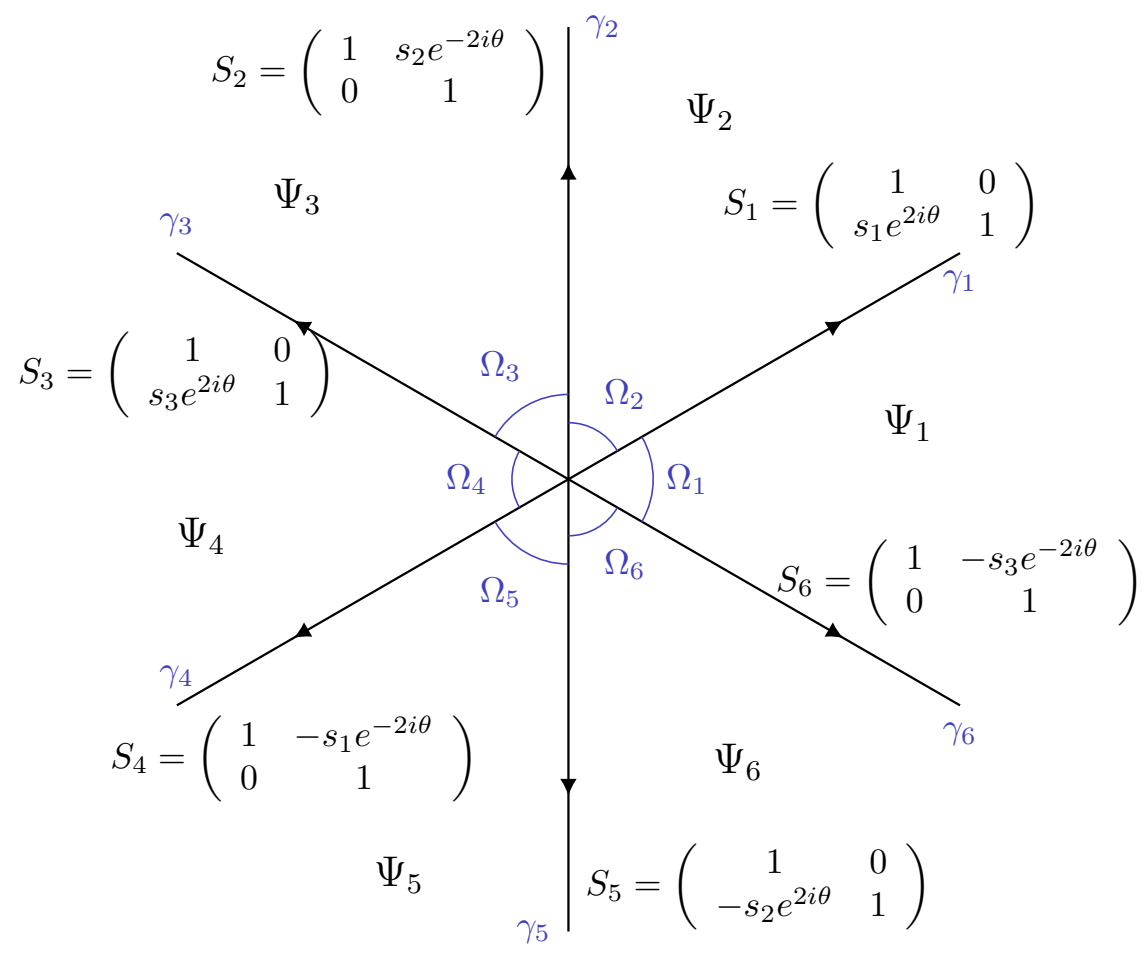

Figure 1: Stokes' rays

The constraint on Stokes' data 2.5 implies that the solution $\Psi(z, t)$ depends only on two Stokes' parameters. We will see in the next subsection that all the functions depend on $s_{1}, s_{3}$. In this paper, we are concerned with the generic 2-parameter solutions that correspond to the following constraints on the Stokes' parameters

$$
s_{1} s_{3} \neq 1 ; \quad \arg \left(1-s_{1} s_{3}\right) \in(-\pi, \pi) .
$$

In order to modify the Riemann-Hilbert contour of Painlevé II, we perform the change of variables

$$
\lambda=(-x)^{1 / 2} z, \quad t=(-x)^{3 / 2} .
$$

The characterstic exponent $\exp (i \theta(\lambda, x))$ in 2.4$)$ is then replaced by

$$
e^{i t \theta(z)}, \quad \theta(z)=\frac{4}{3} z^{3}-z .
$$

The stationary points are then $z_{ \pm}= \pm 1 / 2$. The contour in fig. 1 can be deformed into fig. 2

\footnotetext{
${ }^{2}$ The Ablowitz-Segur family of solutions correspond to the case $s_{2}=0$.
} 


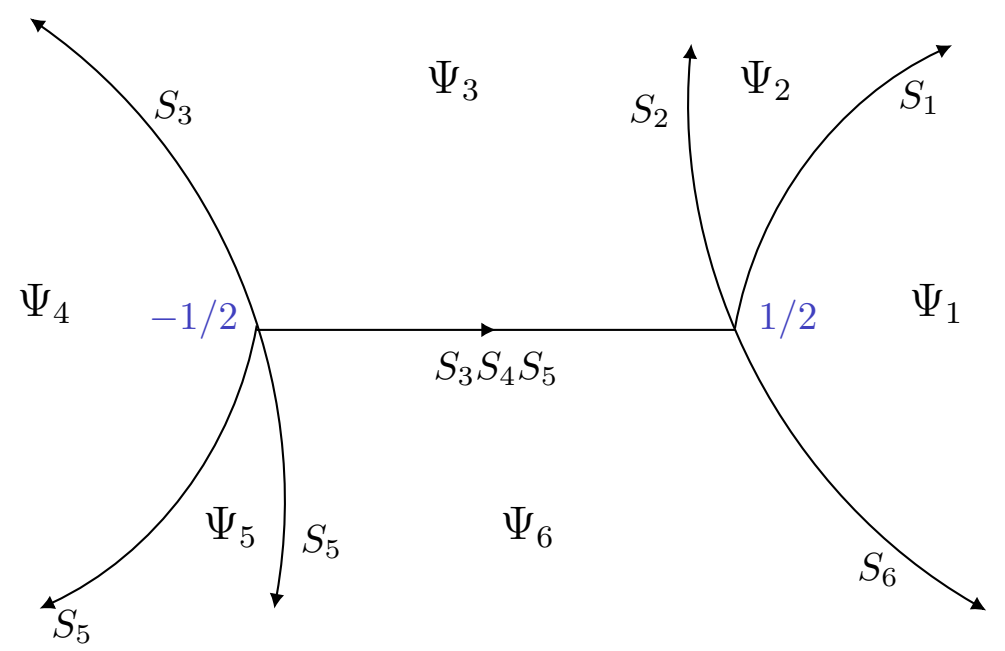

Figure 2: Deforming the contour in fig. 1

Noticing that the product of Stokes' matrices $\left(S_{3} S_{4} S_{5}\right)^{-1}$ can be written as a product of lower triangular, diagonal and upper triangular matrices (LDU)

$$
\begin{gathered}
\left(S_{3} S_{4} S_{5}\right)^{-1}=\left(\begin{array}{cc}
1-s_{1} s_{3} & s_{1} e^{-2 i t \theta(z)} \\
s_{1} e^{2 i t \theta(z)} & 1+s_{1} s_{2}
\end{array}\right)=S_{L} S_{D} S_{U} \\
=\left(\begin{array}{cc}
1 & 0 \\
s_{1}\left(1-s_{1} s_{3}\right)^{-1} e^{2 i t \theta(z)} & 1
\end{array}\right)\left(\begin{array}{cc}
1-s_{1} s_{3} & 0 \\
0 & \left(1-s_{1} s_{3}\right)^{-1}
\end{array}\right)\left(\begin{array}{cc}
1 & s_{1}\left(1-s_{1} s_{3}\right)^{-1} e^{-2 i t \theta(z)} \\
0 & 1
\end{array}\right),
\end{gathered}
$$

the contour in fig. 1 can be transformed into the contour $\Sigma$ in fig. 3. One can easily check there is no monodromy around the points $z= \pm 1 / 2$.

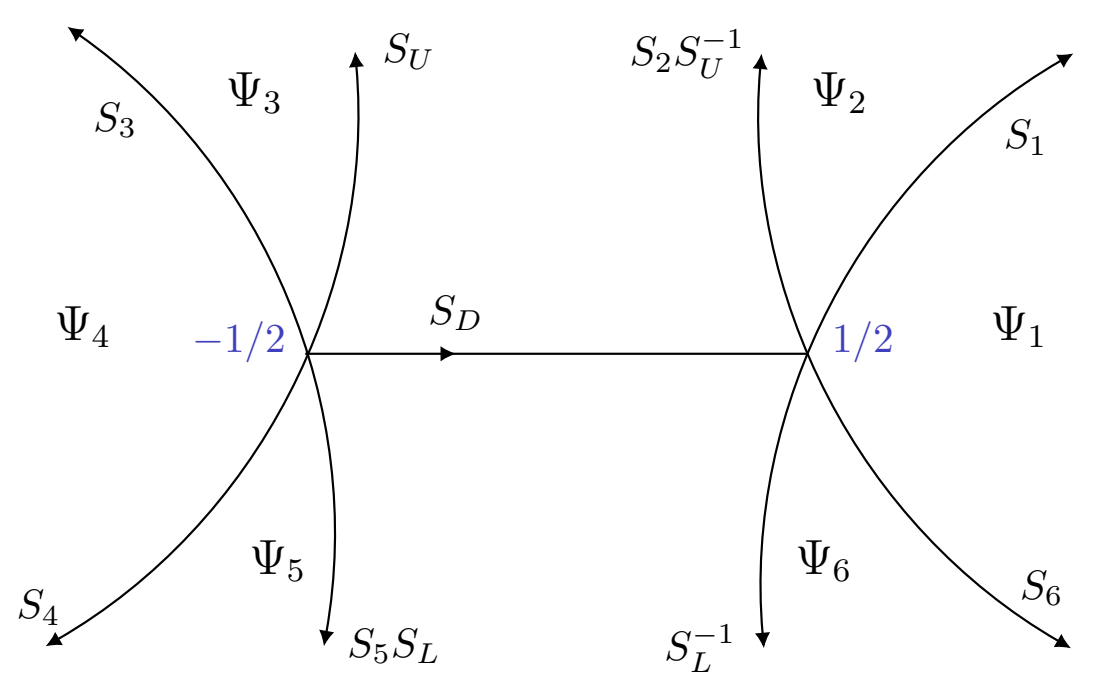

Figure 3: Deformed Painlevé II Riemann-Hilbert contour $\Sigma$.

In fig. 3, $S_{D}=\left(1-s_{1} s_{3}\right)^{\sigma_{3}}$. Defining

$$
\nu=-\frac{1}{2 \pi i} \log \left(1-s_{1} s_{3}\right),
$$


one can verify that the function

$$
\Psi^{D}(z, t)=\left(\frac{z-z_{-}}{z-z_{+}}\right)^{\nu \sigma_{3}}
$$

satisfies the RHP on the segement $\left[z_{-}, z_{+}\right]$where $z_{ \pm}= \pm 1 / 2$. On the contour $\Sigma$ in fig. 3 . the function $\Psi(z, t)$ in 1.2 solves the following RHP

\section{Riemann-Hilbert problem 2.}

- $\Psi(z, t)$ is analytic on $z \in \mathbb{C} \backslash \Sigma$.

- For $z \in \Sigma$, on each of the Stokes' rays

$$
\Psi_{-}^{-1}(z, t) \Psi_{+}(z, t)=G(z, t),
$$

where $G(z, t)$ is piecewise defined on each of the rays of the contour $\Sigma$.

- $\lim _{z \rightarrow \infty} \Psi(z, t)=\mathbb{1}+\mathcal{O}\left(\frac{1}{z}\right)$

In terms of the RHP (2.13), the $\tau$-function 1.6 is

$$
\partial_{t} \log \tau_{P I I} \equiv \partial_{t} \log \tau_{\Sigma}(t):=\int_{\Sigma} \frac{d z}{2 \pi i} \operatorname{Tr}\left[\Psi_{-}^{-1} \Psi_{-}^{\prime} \dot{G} G^{-1}\right]
$$

where $\dot{G}$ means derivative with respect to $t$ and $\Psi_{-}^{\prime}$ means derivative with respect to $z$. We will convert the above expression to a Fredholm determinant in theorem 1 .

\subsection{Parametrices}

To express the $\tau$-function 2.14 in terms of a Fredholm determinant we need to construct a "parametrix" solutions, namely "local solutions" of the RHP. These local solutions are patched together and the actual problem can be recast as the solution of a compact (traceclass) perturbation of the identity.

The effectiveness of the idea relies entirely upon the level of simplicity of these parametrices; the simpler (or rather, more explicit) these reference parametrices are, the more practical the approach is in studying the final problem.

Keeping this in mind, in this section we construct an explicit solution to a RiemannHilbert problem to be used as parametrix for the final one. To this end we recall from ([19], Ch.9 pg.318) the construction of the local parametrices of Painlevé II RHP in fig. 3(the left and right parametrices around the points $z= \pm 1 / 2$ respectively), in terms of parabolic cylinder functions [19].

\subsubsection{Model problem}

Let $Z(\zeta)$ be a $2 \times 2$ matrix valued function that solves the following RHP.

\section{Riemann-Hilbert problem 3.}

- $Z(\zeta)$ is a piecewise holomorphic function defined as follows in each sector shown in fig. 3

$$
Z(\zeta)= \begin{cases}Z_{0}(\zeta), & \arg \zeta \in\left(-\frac{\pi}{4}, 0\right) \\ Z_{1}(\zeta), & \arg \zeta \in\left(0, \frac{\pi}{2}\right) \\ Z_{2}(\zeta), & \arg \zeta \in\left(\frac{\pi}{2}, \pi\right) \\ Z_{3}(\zeta), & \arg \zeta \in\left(\pi, \frac{3 \pi}{2}\right) \\ Z_{4}(\zeta), & \arg \zeta \in\left(\frac{3 \pi}{2}, \frac{7 \pi}{4}\right)\end{cases}
$$


Under the transformation $\zeta \rightarrow-\zeta$ the following symmetry relation holds

$$
\sigma_{3} Z_{k+2}\left(e^{i \pi} \zeta\right) \sigma_{3}=Z_{k}(\zeta) e^{-i \pi(\nu+1) \sigma_{3}} .
$$

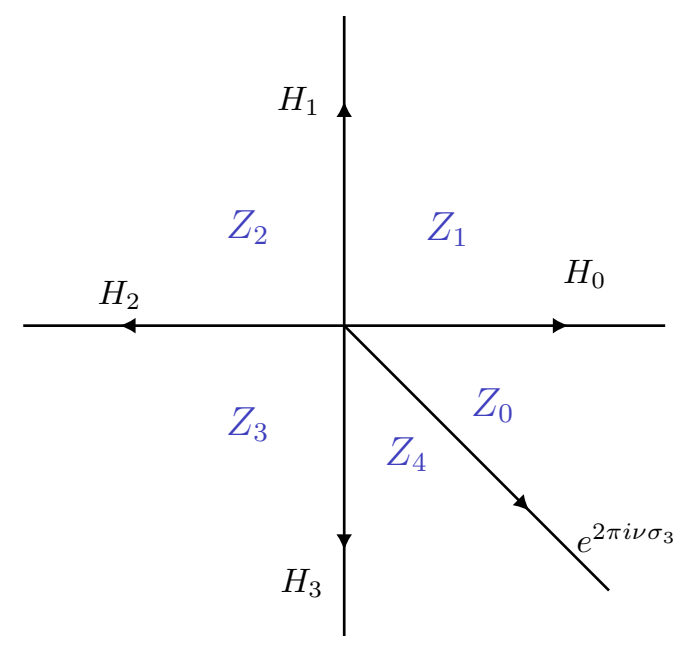

Figure 4: Riemann-Hilbert contour of parabolic cylinder function.

- In each sector, the following jump conditions are satisfied

$$
Z_{k+1}(\zeta)=Z_{k}(\zeta) H_{k}, \quad \arg \zeta=\frac{\pi}{2} k, \quad k=0,1,2,3,4,
$$

and $Z_{5}=Z_{0}$. The jump matrices

$$
\begin{gathered}
H_{0}=\left(\begin{array}{cc}
1 & 0 \\
h_{0} & 1
\end{array}\right), \quad H_{1}=\left(\begin{array}{cc}
1 & h_{1} \\
0 & 1
\end{array}\right), \quad H_{4} \equiv H_{D}=e^{2 \pi i \nu \sigma_{3}} ; \\
H_{k+2}=e^{i \pi\left(\nu+\frac{1}{2}\right) \sigma_{3}} H_{k} e^{-i \pi\left(\nu+\frac{1}{2}\right) \sigma_{3}}, \quad \text { for } k=0,1 .
\end{gathered}
$$

The Stokes' parameters $h_{0}$ and $h_{1}$ are defined as follows

$$
h_{0}=-i \frac{\sqrt{2 \pi}}{\Gamma(\nu+1)}, \quad h_{1}=\frac{\sqrt{2 \pi}}{\Gamma(-\nu)} e^{i \pi \nu}, \quad 1+h_{0} h_{1}=e^{2 \pi i \nu},
$$

and the identity $e^{2 \pi i \nu \sigma_{3}} H_{0} H_{1} H_{2} H_{3}=I$ implies the triviality of the monodromy at the origin.

- As $\zeta \rightarrow \infty$,

$$
Z(\zeta)=\zeta^{-\sigma / 2} \frac{1}{\sqrt{2}}\left(\begin{array}{cc}
1 & 1 \\
1 & -1
\end{array}\right)\left(\mathbb{1}+O\left(\zeta^{-2}\right)\right) e^{\left(\frac{\zeta^{2}}{4}-\left(\nu+\frac{1}{2}\right) \log \zeta\right) \sigma_{3}}
$$

In the zeroth sector, $Z(\zeta)$ is expressed in terms of the Wronskian of the parabolic cylinder functions as

$$
Z_{0}(\zeta)=2^{-\sigma_{3} / 2}\left(\begin{array}{cc}
D_{-\nu-1}(i \zeta) & D_{\nu}(\zeta) \\
\frac{d}{d \zeta} D_{-\nu-1}(i \zeta) & \frac{d}{d \zeta} D_{\nu}(\zeta)
\end{array}\right)\left(\begin{array}{cc}
e^{i \frac{\pi}{2}(\nu+1)} & 0 \\
0 & 1
\end{array}\right)
$$

The parabolic cylinder functions $D_{\nu}(z), D_{-\nu-1}(i z)$ are independent solutions to the following differential equation

$$
\frac{d^{2} y(z)}{d z^{2}}+\left(\nu+\frac{1}{2}-\frac{1}{4} z^{2}\right) y(z)=0 .
$$




\subsubsection{Local parametrices}

Under the conformal map

$$
\zeta(z)=2 \sqrt{-\frac{4 i t}{3} z^{3}+i t z-\frac{i t}{3}}
$$

we define the right parametrix around $z_{+}=1 / 2$ as

$\psi_{r}(z, t)=\left(\zeta(z) \frac{z-z_{-}}{z-z_{+}}\right)^{\nu \sigma_{3}}\left(-\frac{h_{1}}{s_{3}}\right)^{-\sigma_{3} / 2} e^{\frac{i t}{3} \sigma_{3}} 2^{-\sigma_{3} / 2}\left(\begin{array}{cc}\zeta(z) & 1 \\ 1 & 0\end{array}\right) Z(\zeta(z))\left(-\frac{h_{1}}{s_{3}}\right)^{\sigma_{3} / 2} e^{i t \theta(z) \sigma_{3}}$,

and the left parametrix around $z=-1 / 2$ is determined through the symmetry relation

$$
\psi_{l}(z, t)=\sigma_{2} \psi_{r}(-z, t) \sigma_{2} .
$$

In (2.24), the parameter $\nu$ and $h_{1}$ are determined by the Stokes' parameters $s_{1}, s_{3}$. Recall from (2.11) and 2.19),

$$
\nu=-\frac{1}{2 \pi i} \log \left(1-s_{1} s_{3}\right) ; \quad h_{1}=\frac{\sqrt{2 \pi}}{\Gamma(-\nu)} e^{i \pi \nu} .
$$


Figure 5: Mapping the $\zeta$-plane to the right-half of $z$-plane

In each sector,

$\psi_{r}^{(k)}(z, t)=\left(\zeta(z) \frac{z-z_{-}}{z-z_{+}}\right)^{\nu \sigma_{3}}\left(-\frac{h_{1}}{s_{3}}\right)^{-\sigma_{3} / 2} e^{\frac{i t}{3} \sigma_{3}} 2^{-\sigma_{3} / 2}\left(\begin{array}{cc}\zeta(z) & 1 \\ 1 & 0\end{array}\right) Z_{k}(\zeta(z))\left(-\frac{h_{1}}{s_{3}}\right)^{\sigma_{3} / 2} e^{i t \theta(z) \sigma_{3}}$.

The jumps on Stokes' rays in the right and left half planes are denoted by

$$
G_{r}:=\left.G(z, t)\right|_{\Re(z)>0} ; \quad G_{l}:=\left.G(z, t)\right|_{\Re(z)<0} .
$$

As a consequence of (2.25),

$$
G_{l}(z, t)=\sigma_{2} G_{r}(-z, t) \sigma_{2}
$$


We now establish the relation between the Stokes' matrices of the parabolic cylinder functions $H_{i}$ in 2.18$)$ and $G_{r} \equiv G_{r}^{(i)}$ in 2.28$)$. Introducing the notation

$$
h=\left(-\frac{h_{1}}{s_{3}}\right)^{1 / 2},
$$

in a sector $k$ on the right half-plane in fig. 3, $\psi_{r}$ satisfies the following jump condition

$$
\begin{gathered}
\psi_{r}^{k+1}(z, t)=\psi_{r}^{k}(z, t) e^{-i t \theta(z) \sigma_{3}}\left(-\frac{h_{1}}{s_{3}}\right)^{-\sigma_{3} / 2} Z_{k}^{-1} Z_{k+1}\left(-\frac{h_{1}}{s_{3}}\right)^{\sigma_{3} / 2} e^{i t \theta(z) \sigma_{3}} \\
=\psi_{r}^{k}(z, t) e^{-i t \theta(z) \sigma_{3}} h^{-\sigma_{3}} H_{k} h^{\sigma_{3}} e^{i t \theta(z) \sigma_{3}} \\
=\psi_{r}^{k}(z, t) G_{r}^{k} .
\end{gathered}
$$

Note that $Z_{5}=Z_{0}$ implies that $\psi_{r}^{5}(z, t)=\psi_{r}^{0}(z, t)$. Therefore, in terms of $H_{k}, G_{r}$ is

$$
G_{r}^{(k)}(z, t)=e^{-i t \theta \sigma_{3}} h^{-\sigma_{3}} H_{k} h^{\sigma_{3}} e^{i t \theta \sigma_{3}} .
$$

We define the variable

$$
\xi(z, t):=\zeta(-z, t)
$$

that maps the $\xi$-plane to the left half-plane of fig 3 and a similar computation follows for the left parametrix due to the symmetry relation $(2.25)$. We denote the jump condition in each sector on the respective half-planes in fig. 3 by

$$
\psi_{r, l ;+}(z, t)=\psi_{r, l ;-}(z, t) G_{r, l}(z, t) .
$$

Remark 1. The transformation 2.23 is not valid at the point $t=0$. This implies that $\tau_{P I I}$ in 1.8 is valid for $t \in \mathbb{C} \backslash 0$.

\section{Reduction to a RHP along the imaginary axis}

Define a matrix function $\Theta(z, t)$ as a ratio of the global solution $\Psi$ on $\Sigma$ in (2.13) and the local parametrices $\psi_{r}$ in (2.24), $\psi_{l}$ in (2.25).

$$
\Theta(z, t):=\left\{\begin{array}{l}
\Psi(z, t) \psi_{r}^{-1}(z, t) ; \Re(z)>0 \\
\Psi(z, t) \psi_{l}^{-1}(z, t) ; \Re(z)<0 .
\end{array}\right.
$$

Note that the local parametrices cancel the jump of the global parametrix on $\Sigma$, ensuring that the function $\Theta(z, t)$ has a jump only on the imaginary axis, solving the following RHP.

\section{Riemann-Hilbert problem 4.}

- $\Theta(z, t)$ is analyic on $z \in \mathbb{C} \backslash i \mathbb{R}$

- For $z \in i \mathbb{R}$,

$$
\Theta_{+}(z, t)=\Theta_{-}(z, t) J(z, t)
$$

where $J(z, t)=\psi_{r}^{(0)}(z, t)\left[\psi_{l}^{(4)}(z, t)\right]^{-1}$.

- As $z \rightarrow \infty, \Theta(z, t)=\mathbb{1}+\mathcal{O}\left(z^{-1}\right)$. 


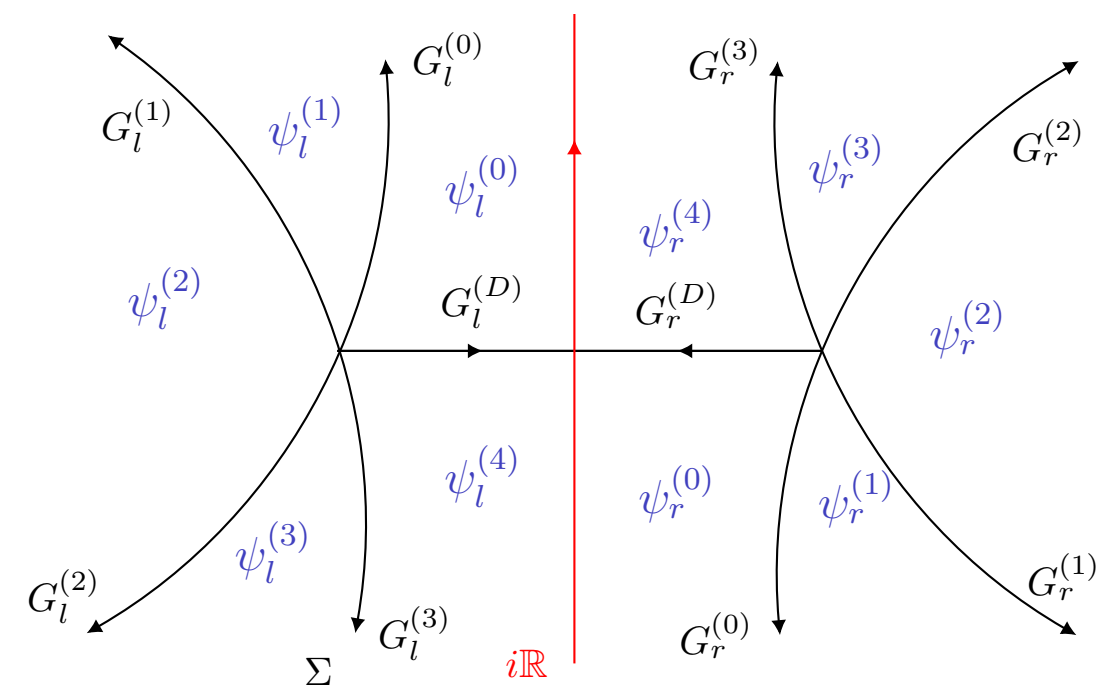

Figure 6: Reducing the Painlevé II RHP on to the imaginary axis.

Remark 2. The solution of the RHP 2 defines, via (3.1) a solution of the RHP 4. Viceversa any solution of the RHP 4 provides a solution to the RHP 2 by means of the inverse of the transformation (3.1). Thus we regard these two problems as equivalent in the sense that the solvability of one of them is necessary and sufficient condition for the solvability of the other.

For later use we compute the expression of the jump matrix $J$ in 3.2 .

Lemma 1. The jump on the imaginary axis

$$
J(z, t)=\Theta_{-}(z, t)^{-1} \Theta_{+}(z, t)=\psi_{r}^{(0)}(z, t)\left[\psi_{l}^{(4)}(z, t)\right]^{-1}=\left(\begin{array}{ll}
\mathcal{A}(z, t) & \mathcal{B}(z, t) \\
\mathcal{C}(z, t) & \mathcal{D}(z, t)
\end{array}\right)
$$

where

$$
\begin{gathered}
\mathcal{A}(z, t)=\frac{1}{h^{4}} \zeta^{\nu} \xi^{\nu} e^{\frac{2 i}{3} t}\left(-e^{-\pi i \nu} h^{4} D_{-\nu}(i \zeta) D_{-\nu}(i \xi)-\nu^{2} e^{2 \pi i \nu} D_{\nu-1}(\zeta) D_{\nu-1}(\xi)\right) \\
\mathcal{B}(z, t)=-\frac{1}{h^{2}}\left(\frac{z-z_{-}}{z-z_{+}}\right)^{2 \nu} \zeta^{\nu} \xi^{-\nu}\left(-i e^{-\pi i \nu} h^{4} D_{-\nu}(i \zeta) D_{-\nu-1}(i \xi)-\nu e^{2 \pi i \nu} D_{\nu-1}(\zeta) D_{\nu}(\xi)\right) \\
\mathcal{C}(z, t)=\mathcal{B}(-z, t) ; \quad \operatorname{det} J=1 .
\end{gathered}
$$

The variables $\zeta \equiv \zeta(z, t), \xi \equiv \xi(z, t)$ are defined in 2.23), 2.33); and $h$ is defined in terms of Stokes' parameters in 2.30.

Proof. Since $\Psi(z, t)$ has no jump on $i \mathbb{R}, J(z, t)$ can be determined solely in terms of $\psi_{r}^{(0)}(z, t)$ and $\psi_{l}^{(4)}(z, t)$. One can check the no monodromy condition at the origin,

$$
\psi_{r}^{(0)}(z, t)\left[\psi_{l}^{(4)}(z, t)\right]^{-1}=\psi_{r}^{(4)}(z, t)\left[\psi_{l}^{(0)}(z, t)\right]^{-1} .
$$

To ease the notation, we define

$$
m(z):=\frac{z-z_{-}}{z-z_{+}}
$$


and observe that the following identities hold

$$
\begin{gathered}
\theta(z)=\frac{4}{3} z^{3}-z=i \frac{\zeta^{2}}{4 t}-\frac{1}{3}=-i \frac{\xi^{2}}{4 t}+\frac{1}{3}, \\
\left(\zeta^{2}+\xi^{2}\right)=-\frac{8 i t}{3} .
\end{gathered}
$$

The function $\psi_{r}^{(0)}(z, t)$ is computed by substituting the zeroth sector solution of the parabolic cylinder function (2.21) in (2.27),

$$
\begin{aligned}
& \psi_{r}^{(0)}(z)=\left(\zeta(z) \frac{z-z_{-}}{z-z_{+}}\right)^{\nu \sigma_{3}}\left(-\frac{h_{1}}{s_{3}}\right)^{-\sigma_{3} / 2} e^{\frac{i t}{3} \sigma_{3}} 2^{-\sigma_{3} / 2}\left(\begin{array}{cc}
\zeta(z) & 1 \\
1 & 0
\end{array}\right) Z_{0}(\zeta(z))\left(-\frac{h_{1}}{s_{3}}\right)^{\sigma_{3} / 2} \times e^{i t \theta(z) \sigma_{3}} \\
& \quad=\left[\begin{array}{cc}
e^{i \pi \nu / 2} e^{-\zeta^{2} / 4} m(z)^{\nu} \zeta^{\nu} D_{-\nu}(i \zeta) & \frac{\nu}{h^{2}} e^{2 i t / 3} e^{\zeta^{2} / 4} m(z)^{\nu} \zeta^{\nu} D_{\nu-1}(\zeta) \\
i h^{2} e^{i \pi \nu / 2} e^{-2 i t / 3} e^{-\zeta^{2} / 4} m(z)^{-\nu} \zeta^{-\nu} D_{-\nu-1}(i \zeta) & e^{\zeta^{2} / 4} m(z)^{-\nu} \zeta^{-\nu} D_{\nu}(\zeta)
\end{array}\right] .
\end{aligned}
$$

The last line is obtained by using (3.7), and the following identity of parabolic cylinder functions

$$
\frac{z}{2} D_{\nu}(z)+D_{\nu}^{\prime}(z)=\nu D_{\nu-1}(z)
$$

The left parametrix $\psi_{l}^{(4)}$ can be obtained in a similar fashion, first by substituting $Z_{4}=$ $Z_{0} e^{-2 i \pi \nu \sigma_{3}}$ from (2.17) in 2.27) to obtain $\psi_{r}^{4}$ and using the relation 2.25 to obtain $\psi_{l}^{(4)}$ as follows

$$
\begin{gathered}
\psi_{l}^{4}(z, t)=\sigma_{2} \psi_{r}^{4}(-z, t) \sigma_{2}=\sigma_{2} \psi_{r}^{0}(-z, t) H_{D}^{-1} \sigma_{2} \\
=\left[\begin{array}{cc}
e^{2 \pi i \nu} e^{\xi^{2} / 4} m(z)^{\nu} \xi^{-\nu} D_{\nu}(\xi) & -i h^{2} e^{-3 \pi i \nu / 2} e^{-2 i t / 3} e^{\xi^{2} / 4} m(z)^{\nu} \xi^{-\nu} D_{-\nu-1}(i \xi) \\
-e^{2 \pi i \nu} \nu h^{-2} e^{2 i t / 3} e^{\xi^{2} / 4} m(z)^{-\nu} \xi^{\nu} D_{\nu-1}(\xi) & e^{-3 \pi i \nu / 2} e^{-y^{2} / 4} m(z)^{-\nu} \xi^{\nu} D_{-\nu}(i \xi)
\end{array}\right] .
\end{gathered}
$$

To obtain the last line, we substitute the expression for $Z_{0}(2.21)$ and simplify the resulting expression using (3.7). Furthermore,

$$
\operatorname{det}\left[\psi_{r}^{(0)}(z, t)\right]=1 ; \quad \operatorname{det}\left[\psi_{l}^{(4)}(z, t)\right]=1
$$

due to the following identity for the Wronskian determinant of parabolic cylinder functions

$$
\mathcal{W}\left[D_{-\nu-1}(i \zeta), D_{\nu}(\zeta)\right]=i e^{-i \pi \nu / 2} .
$$

The jump $J(z, t)$ is then obtained by a straightforward substitution of $(3.8)$ and $(3.10)$ in (3.3), and using (3.7).

$$
J(z, t)=\psi_{r}^{(0)}(z, t)\left[\psi_{l}^{(4)}(z, t)\right]^{-1}=\left(\begin{array}{ll}
\mathcal{A}(z, t) & \mathcal{B}(z, t) \\
\mathcal{C}(z, t) & \mathcal{D}(z, t)
\end{array}\right)
$$


where

$$
\begin{gathered}
\mathcal{A}(z, t)=\zeta^{\nu} \xi^{\nu} e^{\frac{2 i}{3} t}\left(e^{-\pi i \nu} D_{-\nu}(i \zeta) D_{-\nu}(i \xi)+\nu^{2} h^{-4} e^{2 \pi i \nu} D_{\nu-1}(\zeta) D_{\nu-1}(\xi)\right) \\
\mathcal{B}(z, t)=\left(\frac{z-z_{-}}{z-z_{+}}\right)^{2 \nu} \zeta^{\nu} \xi^{-\nu}\left(i h^{2} e^{-i \pi \nu} D_{-\nu}(i \zeta) D_{-\nu-1}(i \xi)+\nu h^{-2} e^{2 \pi i \nu} D_{\nu-1}(\zeta) D_{\nu}(\xi)\right) \\
\mathcal{C}(z, t)=\left(\frac{z-z_{-}}{z-z_{+}}\right)^{-2 \nu} \zeta^{-\nu} \xi^{\nu}\left(i h^{2} e^{-i \pi \nu} D_{-\nu-1}(i \zeta) D_{-\nu}(i \xi)+\nu h^{-2} e^{2 \pi i \nu} D_{\nu}(\zeta) D_{\nu-1}(\xi)\right)=\mathcal{B}(-z, t) \\
\mathcal{D}(z, t)=\zeta^{-\nu} \xi^{-\nu} e^{-\frac{2 i}{3} t}\left(-e^{-\pi i \nu} h^{4} D_{-\nu-1}(i \zeta) D_{-\nu-1}(i \xi)+e^{2 \pi i \nu} D_{\nu}(\zeta) D_{\nu}(\xi)\right)
\end{gathered}
$$

It is obvious that det $J(z, t)=1$. Recall that 2.33$): \xi(-z, t)=\zeta(z, t)$ with $\zeta$ defined in 2.23), $h=\left(-\frac{h_{1}}{s_{3}}\right)^{1 / 2}: 2.30$ where $h_{1}, \nu$ are determined by the Stokes' parameters $s_{1}, s_{3}$ as in 2.19), 2.26 respectively.

The two equivalent RHPs 2, 4 give rise to two corresponding Malgrange forms. Although the two problems are equivalent, the two corresponding tau function may (and in fact do) differ, but only by a non-vanishing term which we now set up to compute. Recalling the Malgrange form of Painlevé II on $\Sigma$ in 2.14):

$$
\partial_{t} \log \tau_{\Sigma}=\int_{\Sigma} \frac{d z}{2 \pi i} \operatorname{Tr}\left[\Psi_{-}^{-1} \Psi_{-}^{\prime} \dot{G} G^{-1}\right] .
$$

Similarly on $i \mathbb{R}$, the RHP 4 satisfies the jump condition $\Theta_{+}=\Theta_{-} J$ and the corresponding Malgrange form (1.6) is

$$
\partial_{t} \log \tau_{i \mathbb{R}}=\int_{i \mathbb{R}} \frac{d z}{2 \pi i} \operatorname{Tr}\left[\Theta_{-}^{-1} \Theta_{-}^{\prime} \dot{J} J^{-1}\right] .
$$

Proposition 1. The Malgrange forms corresponding to the RHPs on the contours $\Sigma$ and $i \mathbb{R}$ are related as

$$
\partial_{t} \log \tau_{\Sigma}=\partial_{t} \log \tau_{i \mathbb{R}}-\int_{i \mathbb{R}} \frac{d z}{2 \pi i} \mathcal{F}(z, t ; \nu, h)-\left[\frac{4 i \nu}{3}+\frac{2 \nu^{2}}{t}\right],
$$

where $\mathcal{F}(z, t ; \nu, h)$ is a regular function explicit in terms of parabolic cylinder functions.

Proof. 4 We begin by computing the expression

$$
\operatorname{Tr}\left\{\Theta_{-}^{-1} \Theta_{-}^{\prime} \dot{J} J^{-1}\right\}
$$

Computing (3.18) term by term using (3.1): $\Theta_{-}=\Psi \psi_{r}^{-1}$,

$$
\begin{gathered}
\Theta_{-}^{-1} \Theta_{-}^{\prime}=\left(\Psi \psi_{r}^{-1}\right)^{-1}\left(\Psi \psi_{r}^{-1}\right)^{\prime}=\psi_{r} \Psi^{-1}\left(\Psi^{\prime} \psi_{r}^{-1}-\Psi \psi_{r}^{-1} \psi_{r}^{\prime} \psi_{r}^{-1}\right) \\
=\psi_{r}\left(\Psi^{-1} \Psi^{\prime}-\psi_{r}^{-1} \psi_{r}^{\prime}\right) \psi_{r}^{-1}
\end{gathered}
$$

Since (3.3): $J=\psi_{r} \psi_{l}^{-1}$,

$$
\begin{gathered}
\dot{J} J^{-1}=\frac{\partial}{\partial t}\left(\psi_{r} \psi_{l}^{-1}\right)\left(\psi_{r} \psi_{l}^{-1}\right)^{-1}=\left(\dot{\psi}_{r} \psi_{l}^{-1}-\psi_{r} \psi_{l}^{-1} \dot{\psi}_{l} \psi_{l}^{-1}\right) \psi_{l} \psi_{r}^{-1} \\
=-\psi_{r} \Delta\left(\psi^{-1} \dot{\psi}\right) \psi_{r}^{-1}
\end{gathered}
$$

\footnotetext{
${ }^{4}$ In the proof, we drop the $z, t$ dependence for the ease of writing. All the functions here on depend on $z, t$ unless specified.
} 
where

$$
\Delta\left(\psi^{-1} \dot{\psi}\right)=\psi_{l}^{-1} \dot{\psi}_{l}-\psi_{r}^{-1} \dot{\psi}_{r}
$$

Substituting 3.19 and 3.20 in 3.18 and using cyclicity of trace,

$$
\operatorname{Tr}\left\{\Theta_{-}^{-1} \Theta_{-}^{\prime} \dot{J} J^{-1}\right\}=\operatorname{Tr}\left\{\left(-\Psi^{-1} \Psi^{\prime}+\psi_{r}^{-1} \psi_{r}^{\prime}\right) \Delta\left(\psi^{-1} \dot{\psi}\right)\right\} .
$$

Since the term $\psi_{r}^{-1} \psi_{r}^{\prime} \Delta\left(\psi^{-1} \dot{\psi}\right)$ is integrated on $i \mathbb{R}$ in 3.16 ,

$\int_{i \mathbb{R}} \frac{d z}{2 \pi i} \operatorname{Tr}\left[\psi_{r}^{-1} \psi_{r}^{\prime} \Delta\left(\psi^{-1} \dot{\psi}\right)\right]=\int_{i \mathbb{R}} \frac{d z}{2 \pi i} \operatorname{Tr}\left[\left(\psi_{r}^{(0)}\right)^{-1}\left(\psi_{r}^{(0)}\right)^{\prime}\left\{\left(\psi_{l}^{(4)}\right)^{-1} \dot{\psi}_{l}{ }^{(4)}-\left(\psi_{r}^{(0)}\right)^{-1} \dot{\psi}_{r}{ }^{(0)}\right\}\right]$

with $\psi_{r}^{(0)}$ defined in 3.8$), \psi_{l}^{(4)}$ in 3.10 . We collect the explicit terms and compute them in the end. Since $\Psi$ has no jump on $i \mathbb{R}$, using Cauchy theorem

$$
\begin{gathered}
-\int_{i \mathbb{R}} \frac{d z}{2 \pi i} \operatorname{Tr}\left\{\Psi^{-1} \Psi^{\prime} \Delta\left(\psi^{-1} \dot{\psi}\right)\right\}=-\int_{i \mathbb{R}} \frac{d z}{2 \pi i} \operatorname{Tr} \Delta\left(\Psi^{-1} \Psi^{\prime}\left(\psi^{-1} \dot{\psi}\right)\right) \\
=\int_{\Sigma} \frac{d z}{2 \pi i} \operatorname{Tr} \Delta\left(\Psi^{-1} \Psi^{\prime}\left(\psi^{-1} \dot{\psi}\right)\right) \\
=\int_{\Sigma_{L}} \frac{d z}{2 \pi i} \operatorname{Tr} \Delta\left(\Psi^{-1} \Psi^{\prime}\left(\psi^{-1} \dot{\psi}\right)\right)+\int_{\Sigma_{R}} \frac{d z}{2 \pi i} \operatorname{Tr} \Delta\left(\Psi^{-1} \Psi^{\prime}\left(\psi^{-1} \dot{\psi}\right)\right),
\end{gathered}
$$

where $\Sigma_{L, R}$ are $\Sigma$ restricted to the left and right half-planes respectively. Since $\Psi$ has jumps on $\Sigma_{L}$,

$$
\int_{\Sigma_{L}} \frac{d z}{2 \pi i} \operatorname{Tr} \Delta\left(\Psi^{-1} \Psi^{\prime}\left(\psi^{-1} \dot{\psi}\right)\right)=\int_{\Sigma_{L}} \frac{d z}{2 \pi i} \operatorname{Tr}\left\{\Psi_{+}^{-1} \Psi_{+}^{\prime}\left(\psi_{l+}^{-1} \dot{\psi_{l+}}\right)-\Psi_{-}^{-1} \Psi_{-}^{\prime}\left(\psi_{l-}^{-1} \dot{\psi_{l-}}\right)\right\} .
$$

similarly on $\Sigma_{R}$

$$
\int_{\Sigma_{R}} \frac{d z}{2 \pi i} \operatorname{Tr} \Delta\left(\Psi^{-1} \Psi^{\prime}\left(\psi^{-1} \dot{\psi}\right)\right)=\int_{\Sigma_{R}} \frac{d z}{2 \pi i} \operatorname{Tr}\left\{\Psi_{+}^{-1} \Psi_{+}^{\prime}\left(\psi_{r+}^{-1} \dot{\psi_{r+}}\right)-\Psi_{-}^{-1} \Psi_{-}^{\prime}\left(\psi_{r-}^{-1} \dot{\psi_{r-}}\right)\right\} .
$$

In order to estimate (3.23), we begin by computing the integrand on $\Sigma_{L}$. Computing (3.24) term by term using 2.13): $\Psi_{+}=\Psi_{-} G_{l}$,

$$
\begin{gathered}
\Psi_{+}^{-1} \Psi_{+}^{\prime}=\left(\Psi_{-} G_{l}\right)^{-1}\left(\Psi_{-} G_{l}\right)^{\prime}=G_{l}^{-1} \Psi_{-}^{-1}\left(\Psi_{-}^{\prime} G_{l}+\Psi_{-} G_{l}^{\prime}\right) \\
=G_{l}^{-1}\left(\Psi_{-}^{-1} \Psi_{-}^{\prime}+G_{l}^{\prime} G_{l}^{-1}\right) G_{l} .
\end{gathered}
$$

Since 2.34): $\psi_{l+}=\psi_{l-} G_{l}$,

$$
\begin{gathered}
\psi_{l+}^{-1} \dot{\psi_{l+}}=G_{l}^{-1} \psi_{l-}^{-1}\left(\dot{\dot{\psi}_{l-}} G_{l}+\psi_{l-} \dot{G}_{l}\right) \\
=G_{l}^{-1}\left(\psi_{l-}^{-1} \dot{\psi_{l-}}+\dot{G}_{l} G_{l}^{-1}\right) G_{l} .
\end{gathered}
$$

The product of (3.26) and (3.27) under the trace reads

$$
\operatorname{Tr}\left\{\Psi_{+}^{-1} \Psi_{+}^{\prime}\left(\dot{\psi_{l+}} \psi_{l+}^{-1}\right)\right\}=\operatorname{Tr}\left[\left(\Psi_{-}^{-1} \Psi_{-}^{\prime}+G_{l}^{\prime} G_{l}^{-1}\right)\left(\psi_{l-}^{-1} \dot{\psi_{l-}}+\dot{G}_{l} G_{l}^{-1}\right)\right] .
$$


Substituting (3.28) in 3.24),

$$
\operatorname{Tr} \Delta\left(\Psi^{-1} \Psi^{\prime}\left(\dot{\psi} \psi^{-1}\right)\right)=\operatorname{Tr}\left[\Psi_{-}^{-1} \Psi_{-}^{\prime} \dot{G}_{l} G_{l}^{-1}+G_{l}^{\prime} G_{l}^{-1}\left(\psi_{l-}^{-1} \dot{\psi}_{l-}+\dot{G}_{l} G_{l}^{-1}\right)\right]
$$

A parallel computation for $\Sigma_{R}$ gives

$$
\operatorname{Tr} \Delta\left(\Psi^{-1} \Psi^{\prime}\left(\dot{\psi} \psi^{-1}\right)\right)=\operatorname{Tr}\left[\Psi_{-}^{-1} \Psi_{-}^{\prime} \dot{G}_{r} G_{r}^{-1}+G_{r}^{\prime} G_{r}^{-1}\left(\psi_{r-}^{-1} \dot{\psi}_{r-}+\dot{G}_{r} G_{r}^{-1}\right)\right] .
$$

Summing the terms $(3.29)$ and $(3.30)$, we obtain that

$$
\begin{gathered}
\partial_{t} \log \tau_{i \mathbb{R}}=\int_{i \mathbb{R}} \operatorname{Tr}\left[\Theta_{-}^{-1} \Theta_{-}^{\prime} \dot{J} J^{-1}\right]=\int_{\Sigma_{R}} \operatorname{Tr}\left[\Psi_{-}^{-1} \Psi_{-}^{\prime} \dot{G}_{r} G_{r}^{-1}\right]+\int_{\Sigma_{L}} \operatorname{Tr}\left[\Psi_{-}^{-1} \Psi_{-}^{\prime} \dot{G}_{l} G_{l}^{-1}\right] \\
+\int_{\Sigma_{L}} \operatorname{Tr}\left[G_{l}^{\prime} G_{l}^{-1}\left(\psi_{l-}^{-1} \dot{\psi_{l-}}+\dot{G}_{l} G_{l}^{-1}\right)\right]+\int_{\Sigma_{R}} \operatorname{Tr}\left[G_{r}^{\prime} G_{r}^{-1}\left(\psi_{r-}^{-1} \dot{\psi_{r-}}+\dot{G}_{r} G_{r}^{-1}\right)\right] \\
+\int_{i \mathbb{R}} \operatorname{Tr}\left[\psi_{r}^{-1} \psi_{r}^{\prime} \Delta\left(\psi^{-1} \dot{\psi}\right)\right] \\
=\int_{\Sigma} \operatorname{Tr}\left[\Psi_{-}^{-1} \Psi_{-}^{\prime} \dot{G} G^{-1}\right]+\int_{i \mathbb{R}} \operatorname{Tr}\left[\psi_{r}^{-1} \psi_{r}^{\prime} \Delta\left(\psi^{-1} \dot{\psi}\right)\right] \\
+\int_{\Sigma_{L}} \operatorname{Tr}\left[G_{l}^{\prime} G_{l}^{-1}\left(\psi_{l-}^{-1} \dot{\psi_{l-}}+\dot{G}_{l} G_{l}^{-1}\right)\right]+\int_{\Sigma_{R}} \operatorname{Tr}\left[G_{r}^{\prime} G_{r}^{-1}\left(\psi_{r-}^{-1} \dot{\psi_{r-}}+\dot{G}_{r} G_{r}^{-1}\right)\right] \\
=\partial_{t} \log \tau_{\Sigma}+\int_{i \mathbb{R}} \operatorname{Tr}\left[\psi_{r}^{-1} \psi_{r}^{\prime} \Delta\left(\psi^{-1} \dot{\psi}\right)\right] \\
+\int_{\Sigma_{L}} \operatorname{Tr}\left[G_{l}^{\prime} G_{l}^{-1}\left(\psi_{l-}^{-1} \dot{\psi_{l-}}+\dot{G}_{l} G_{l}^{-1}\right)\right]+\int_{\Sigma_{R}} \operatorname{Tr}\left[G_{r}^{\prime} G_{r}^{-1}\left(\psi_{r-}^{-1} \dot{\psi_{r-}}+\dot{G}_{r} G_{r}^{-1}\right)\right] .
\end{gathered}
$$

Notice that $\psi_{r, l}$ and $G_{r, l}$ are completely determined in terms of parabolic cylinder functions. The final expression is

$$
\begin{gathered}
\partial_{t} \log \tau_{\Sigma}=\partial_{t} \log \tau_{i \mathbb{R}}-\int_{i \mathbb{R}} \operatorname{Tr}\left[\psi_{r}^{-1} \psi_{r}^{\prime} \Delta\left(\psi^{-1} \dot{\psi}\right)\right] \\
-\int_{\Sigma_{L}} \operatorname{Tr}\left[G_{l}^{\prime} G_{l}^{-1}\left(\psi_{l-}^{-1} \dot{\psi_{l-}}+\dot{G}_{l} G_{l}^{-1}\right)\right]-\int_{\Sigma_{R}} \operatorname{Tr}\left[G_{r}^{\prime} G_{r}^{-1}\left(\psi_{r-}^{-1} \dot{\psi_{r-}}+\dot{G}_{r} G_{r}^{-1}\right)\right] .
\end{gathered}
$$

The following can be said about the explicit terms in (3.32).

- We can completely determine the integrals on $\Sigma_{R, L}$. The symmetry relations 2.25 , 2.29 imply that

$$
\int_{\Sigma_{L}} \operatorname{Tr}\left[G_{l}^{\prime} G_{l}^{-1}\left(\psi_{l-}^{-1} \dot{\psi_{l-}}+\dot{G}_{l} G_{l}^{-1}\right)\right]=\int_{\Sigma_{R}} \operatorname{Tr}\left[G_{r}^{\prime} G_{r}^{-1}\left(\psi_{r-}^{-1} \dot{\psi}_{r-}+\dot{G}_{r} G_{r}^{-1}\right)\right] .
$$

Furthermore, (2.19) implies that the jump $G_{r}^{(k)}$ in 2.32 is lower triangular for $k=0,2$; upper triangular for $k=1,3$; diagonal and constant for $k=4$. Therefore,

$$
\operatorname{Tr}\left[G_{r}^{\prime} G_{r}^{-1} \dot{G}_{r} G_{r}^{-1}\right]=\operatorname{Tr}\left[G_{l}^{\prime} G_{l}^{-1} \dot{G}_{l} G_{l}^{-1}\right]=0 .
$$

We now proceed to compute the following term in 3.32

$$
\int_{\Sigma_{R}} \operatorname{Tr}\left[G_{r}^{\prime} G_{r}^{-1} \psi_{r-}^{-1} \dot{\psi_{r-}}\right]=\sum_{k=1}^{5} \int_{\Sigma_{k}} \frac{d z}{2 \pi i} \operatorname{Tr}\left[\left(G_{r}^{(k)}\right)^{\prime}\left(G_{r}^{(k)}\right)^{-1}\left(\psi_{r-}^{(k-1)}\right)^{-1}{\dot{r_{r-}}}^{(k-1)}\right] .
$$


In each sector, $\psi_{r}$ and $G_{r}$ can be computed starting from $\psi_{r}^{(0)}$ in (3.8), and the jumps in 2.32). A lengthy but straighforward computation yields

$$
\int_{\Sigma_{R}} \operatorname{Tr}\left[G_{r}^{\prime} G_{r}^{-1}\left(\psi_{r-}^{-1} \dot{\psi_{r-}}+\dot{G}_{r} G_{r}^{-1}\right)\right]=\left[\frac{2 i \nu}{3}+\frac{\nu^{2}}{t}\right] .
$$

The relation (3.33) then implies,

$$
\int_{\Sigma_{L}} \operatorname{Tr}\left[G_{l}^{\prime} G_{l}^{-1}\left(\psi_{l-}^{-1} \dot{\psi_{l-}}+\dot{G}_{l} G_{l}^{-1}\right)\right]+\int_{\Sigma_{R}} \operatorname{Tr}\left[G_{r}^{\prime} G_{r}^{-1}\left(\psi_{r-}^{-1} \dot{\psi_{r-}}+\dot{G}_{r} G_{r}^{-1}\right)\right]=\left[\frac{4 i \nu}{3}+\frac{2 \nu^{2}}{t}\right] .
$$

- The remaining explicit term in 3.32

$$
\int_{i \mathbb{R}} \operatorname{Tr}\left[\psi_{r}^{-1} \psi_{r}^{\prime} \Delta\left(\psi^{-1} \dot{\psi}\right)\right] \equiv \int_{i \mathbb{R}} \frac{d z}{2 \pi i} \operatorname{Tr}\left[\left(\psi_{r}^{(0)}\right)^{-1}\left(\psi_{r}^{(0)}\right)^{\prime}\left\{\left(\psi_{l}^{(4)}\right)^{-1} \dot{\psi}_{l}^{(4)}-\left(\psi_{r}^{(0)}\right)^{-1} \dot{\psi}_{r}^{(0)}\right\}\right] \text {. }
$$

The functions $\psi_{r}^{(0)}$ and $\psi_{l}^{(4)}$ depend on $z$ through $\zeta(z, t)$ as in 2.23 and $\xi(z, t)$ as in (2.33) respectively. In order to solve the integral, we need to compute integrals of the form

$$
\int \frac{d z}{2 \pi i} D_{\nu}(\zeta) D_{\mu}(\xi) D_{-\rho}(i \zeta) D_{-\sigma}(i \xi)
$$

which is not exactly solvable. The expression (3.38) is however, explicit. Defining a function $\widetilde{\mathcal{F}}$ as

$$
\widetilde{\mathcal{F}}(z, t ; \nu, h):=\operatorname{Tr}\left[\psi_{r}^{-1} \psi_{r}^{\prime}\left(\psi_{l}^{-1} \dot{\psi}_{l}-\psi_{r}^{-1} \dot{\psi}_{r}\right)\right],
$$

The final expression in 3.32 reads

$$
\partial_{t} \log \tau_{\Sigma}=\partial_{t} \log \tau_{i \mathbb{R}}-\int_{i \mathbb{R}} \frac{d z}{2 \pi i} \widetilde{\mathcal{F}}(z, t ; \nu, h)-\left[\frac{4 i \nu}{3}+\frac{2 \nu^{2}}{t}\right] .
$$

\section{Integrable kernel and Fredholm determinant}

Up to this point, we started with the RHP of Painleve II in fig. 3, used the description of the local parametrices in terms of parabolic cylinder functions in the subsection 2.1 to define a RHP on $i \mathbb{R}(3.2)$ in section 3. We then showed that the corresponding Malgrange forms are related in proposition 1 1 . Our goal now reduces to expressing $\tau_{i \mathbb{R}}$ as a Fredholm determinant.

It is known that a jump $J(z, t) \in S L(2, \mathbb{C})$ on non-intersecting contours can be expressed in terms of lower and upper triangular matrices called the LULU decomposition and the corresponding $\tau$-function can then be written as a Fredholm determinant of an Integrable operator [2]. Here, we modify the construction in [2] by using LDU decomposition instead, which then gives us a simpler kernel. In this section, we

1. transform RHP 4 on to a set of two parallel lines with lower and upper triangular jumps using the LDU decomposition,

2. formulate the $\tau$-function on the set of parallel lines, call it $\tau_{L U}$ as a Fredholm determinant of an integrable operator, and

3. prove that the Malgrange forms on the contours LU and $i \mathbb{R}$ coincide.

\footnotetext{
${ }^{5}$ The author thanks A.Its for suggesting LDU decomposition.
} 


\subsection{LU decomposition}

The RHP on $i \mathbb{R}$ can be transformed on to a set of two parallel lines with jumps that are upper and lower triangular respectively. We decompose the jump $J(z, t)(3.3)$ into lower, diagonal and upper triangular matrices, called the LDU decomposition [15], which recasts the RHP 4 on to a set of three parallel lines.

$$
\begin{aligned}
& J(z, t)=\left(\begin{array}{ll}
\mathcal{A}(z, t) & \mathcal{B}(z, t) \\
\mathcal{C}(z, t) & \mathcal{D}(z, t)
\end{array}\right)=\left(\begin{array}{cc}
1 & 0 \\
\mathcal{C}(z, t) & 1
\end{array}\right)\left(\begin{array}{cc}
\mathcal{A}(z, t) & 0 \\
\mathcal{A}(z, t) & 1
\end{array}\right)\left(\begin{array}{cc}
1 & \frac{\mathcal{B}(z, t)}{\mathcal{A}(z, t)} \\
0 & 1
\end{array}\right) \\
& :=F_{1}(z, t) F_{2}(z, t) F_{3}(z, t) .
\end{aligned}
$$

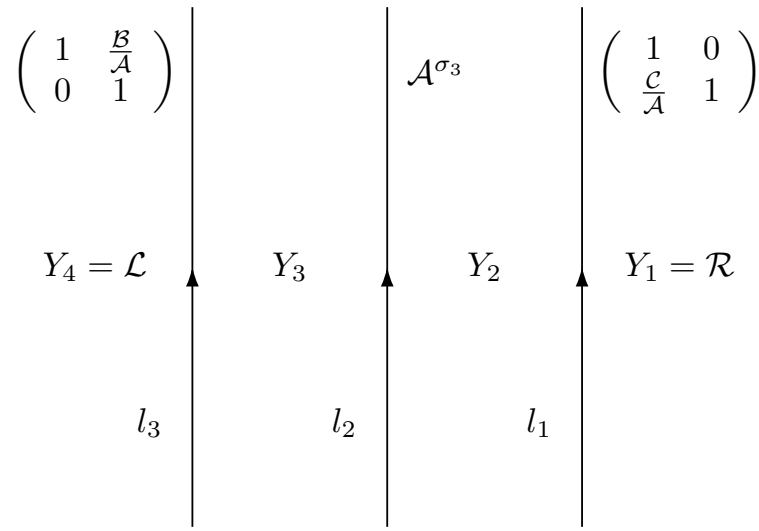

Figure 7: LDU decomposition

The function $Y(z, t)$ then solves the following RHP.

\section{Riemann-Hilbert problem 5.}

- $Y(z, t)$ is a piecewise analytic in $\mathbb{C} \backslash\left(\cup_{i=1}^{3} l_{i}\right)$.

- On each line $l_{i}$ in fig. 7, the following jump condition holds

$$
Y_{i+1}(z, t)=Y_{i}(z, t) F_{i}(z, t),
$$

with the identification

$$
Y_{4}(z, t)=\Theta_{+}(z, t) ; \quad Y_{1}(z, t)=\Theta_{-}(z, t) .
$$

$\Theta_{ \pm}$are defined in (3.1).

- $\lim _{z \rightarrow \infty} Y(z, t)=\mathbb{1}+\mathcal{O}\left(z^{-1}\right)$.

The RHP:5 can be further transformed with the observation that the function $\varphi(z, t)^{\sigma_{3}}$ defined as

$$
\varphi(z, t):=\exp \left[\int_{i \mathbb{R}} \frac{d w}{2 \pi i} \frac{\log \mathcal{A}(w, t)}{z-w}\right],
$$

solves RHP on $l_{2}$ with the diagonal jump $\mathcal{A}^{\sigma_{3}}$ locally with $\mathcal{A}$ defined in (3.4). The ratio of $Y(z, t), \varphi(z, t)^{\sigma_{3}}$

$$
\tilde{Y}_{i}(z, t):=Y_{i}(z, t) \varphi(z, t)^{-\sigma_{3}}
$$

is such that $\widetilde{Y}(z, t)$ jumps only on $l_{1} \cup l_{3}$ and solves the following RHP.

\section{Riemann-Hilbert problem 6.}


- $\tilde{Y}(z, t)$ is piecewise analytic in $\mathbb{C} \backslash\left(l_{1} \cup l_{3}\right)$.

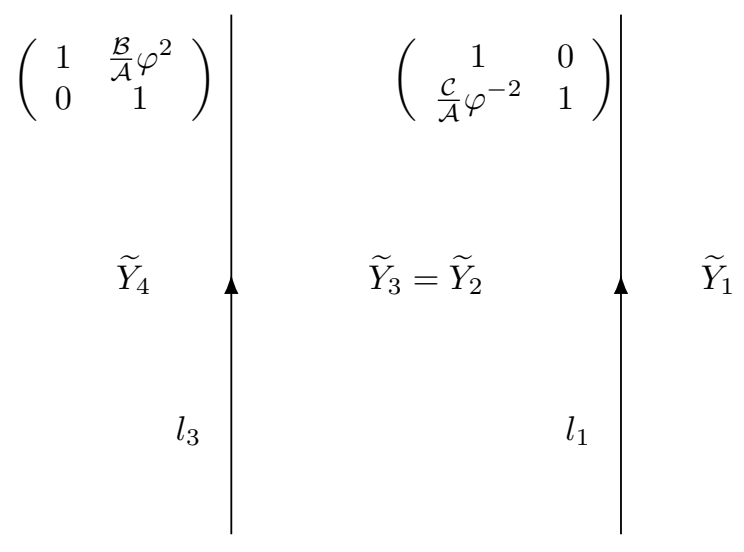

Figure 8: RHP with lower and upper triangular jumps.

- The following jump conditions are valid on the contours $l_{i}, i=1,3$

$$
\widetilde{Y}_{i+1}(z, t)=\tilde{Y}_{i}(z, t) \widetilde{F}_{i}(z, t)
$$

where

$$
\widetilde{F}_{1}(z, t)=\left(\begin{array}{cc}
1 & 0 \\
\frac{\mathcal{C}(z, t)}{\mathcal{A}(z, t)} \varphi(z, t)^{2} & 1
\end{array}\right) ; \quad \widetilde{F}_{3}(z, t)=\left(\begin{array}{cc}
1 & \frac{\mathcal{B}(z, t)}{\mathcal{A}(z, t)} \varphi(z, t)^{-2} \\
0 & 1
\end{array}\right) .
$$

Note that $\tilde{Y}(z, t)$ has no jump on $l_{2}$, implying that $\widetilde{Y}_{3}(z, t)=\widetilde{Y}_{2}(z, t)$.

The RHP 6 in fig. 8 is of the 'integrable' type and its solvability is determined by the invertibility of an integrable operator i.e, its $\tau$-function is the Fredholm determinant of an integrable operator.

\subsection{Integrable kernel}

Proposition 2. The $\tau$-function on $l_{1} \cup l_{3}$ denoted by $\tau_{L U}$ is a Fredholm determinant of an integrable operator

$$
\tau_{L U}=\operatorname{det}\left[\mathbb{1}_{L^{2}(i \mathbb{R})}-\tilde{\mathcal{K}}\right]
$$

where

$$
(\widetilde{\mathcal{K}} h)(z)=\frac{\mathcal{C}(z, t)}{\mathcal{A}(z, t)} \int_{i \mathbb{R}} \frac{d w}{2 \pi i} \int_{i \mathbb{R}+\epsilon} \frac{d \widetilde{w}}{2 \pi i} \frac{\varphi_{+}^{2}(w) \varphi_{+}^{-2}(\widetilde{w})}{(z-\widetilde{w})(\widetilde{w}-w)} \mathcal{A}(\widetilde{w}, t) \mathcal{B}(\widetilde{w}, t) h(\widetilde{w}) .
$$

The functions $\mathcal{A}, \mathcal{B}, \mathcal{C}$ are defined in (3.4) and $\varphi_{+}$is the positive (left of the imaginary axis) boundary value of 4.4.

Proof. Let us recall the jumps in 4.7

$$
\widetilde{F}(z, t)=\left\{\begin{array}{l}
\widetilde{F}_{1}(z, t)=\left(\begin{array}{cc}
1 & 0 \\
\frac{\mathcal{C}(z, t)}{\mathcal{A}(z, t)} \varphi(z, t)^{2} & 1
\end{array}\right) ; \text { on } l_{1} \\
\widetilde{F}_{3}(z, t)=\left(\begin{array}{cc}
1 & \frac{\mathcal{B}(z, t)}{\mathcal{A}(z, t)} \varphi(z, t)^{-2} \\
0 & 1
\end{array}\right) ; \text { on } l_{3}
\end{array}\right.
$$


We define the functions

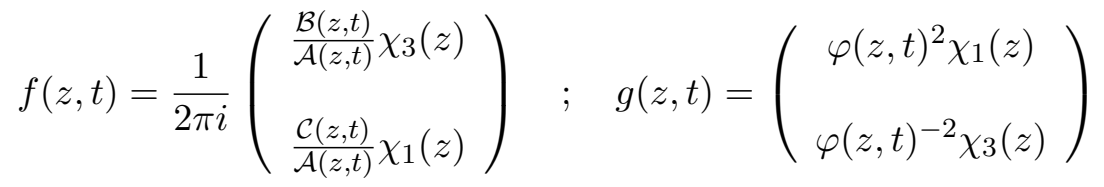

where $\chi_{1}(z), \chi_{3}(z)$ denote the characterstic functions on the contours $l_{1}, l_{3}$ respectively. The jump $F(z, t)$ can be written in terms of 4.11 as

$$
\widetilde{F}=1-2 \pi i f(z) g^{T}(z),
$$

and clearly $f^{T}(z) g(z)=0$. The associated integrable kernel is then

$$
\begin{gathered}
K(z, w)=\frac{f^{T}(z) g(w)}{z-w}= \\
=\frac{1}{z-w}\left(\begin{array}{ll}
\chi_{1}(z) & \chi_{3}(z)
\end{array}\right)\left(\begin{array}{cc}
0 & \frac{\mathcal{C}(z, t)}{\mathcal{A}(z, t)} \varphi^{-2}(w, t) \\
\frac{\mathcal{B}(z, t)}{\mathcal{A}(z, t)} \varphi^{2}(w, t) & 0
\end{array}\right)\left(\begin{array}{l}
\chi_{1}(w) \\
\chi_{3}(w)
\end{array}\right) \\
\equiv\left(\begin{array}{lll}
\chi_{1}(z) & \chi_{3}(z)
\end{array}\right)\left(\begin{array}{cc}
0 & K_{31}(z, w) \\
K_{13}(z, w) & 0
\end{array}\right)\left(\begin{array}{l}
\chi_{1}(w) \\
\chi_{3}(w)
\end{array}\right) .
\end{gathered}
$$

The kernels $K_{13}(z, w)$ and $K_{31}(z, w)$ in 4.13 take the form

$$
\begin{aligned}
K_{13}(z, w) & =\frac{\mathcal{B}(z, t) \varphi^{2}(w, t)}{(2 \pi i) \mathcal{A}(z, t)(z-w)} \\
K_{31}(z, w) & =\frac{\mathcal{C}(z, t) \varphi^{-2}(w, t)}{(2 \pi i) \mathcal{A}(z, t)(z-w)} .
\end{aligned}
$$

We introduce the operators

$$
\begin{gathered}
\mathcal{K}_{31}: L^{2}\left(l_{3}\right) \rightarrow L^{2}\left(l_{1}\right) \\
\mathcal{K}_{13}: L^{2}\left(l_{1}\right) \rightarrow L^{2}\left(l_{3}\right),
\end{gathered}
$$

defined as

$$
\begin{aligned}
& \left(\mathcal{K}_{31} h\right)(z)=\int_{l_{3}} K_{31}(z, w) h(w) d w \\
& \left(\mathcal{K}_{13} \widetilde{h}\right)(z)=\int_{l_{1}} K_{13}(z, w) \widetilde{h}(w) d w .
\end{aligned}
$$

The $\tau$-function corresponding to the RHP 6 is then

$$
\tau_{L U}(t)=\operatorname{det}\left[\mathbb{1}_{L^{2}\left(l_{1} \cup l_{3}\right)}-\left(\begin{array}{cc}
0 & \mathcal{K}_{31} \\
\mathcal{K}_{13} & 0
\end{array}\right)\right] .
$$

Since $\varphi^{2}(w, t)$ is analytic in $\Re(w)>0$ and $\lim _{w \rightarrow \infty} \varphi(w, t)=1, \mathcal{K}_{13}, \mathcal{K}_{31}$ are Traceclass. Therefore we can write $\tau_{L U}(t)$ in the form

$$
\tau_{L U}(t)=\operatorname{det}\left[\mathbb{1}_{L^{2}\left(l_{3}\right)}-\mathcal{K}_{13} \circ \mathcal{K}_{31}\right] .
$$

The form of the $\tau$-function 4.17) can be further modified such that the operator acts on $L^{2}(i \mathbb{R})$ instead of $L^{2}\left(l_{3}\right)$. We begin by splitting the function $h(z)$ as

$$
h(z)=h_{L}(z)+h_{R}(z)
$$


where $h_{L, R}(z)$ are analytic to the left and right of $l_{3}$ respectively, and $h_{L, R}(z)=\mathcal{O}\left(z^{-1}\right)$ as $z \rightarrow \infty$. The integrable operator (4.15) acts on $h(z)$ as

$$
\left(\mathcal{K}_{13} \mathcal{K}_{31} h_{R}\right)(z) \equiv 0 \Rightarrow\left(\mathcal{K}_{13} \mathcal{K}_{31} h\right)(z)=\left(\mathcal{K}_{13} \mathcal{K}_{31} h_{L}\right)(z)
$$

We can therefore move the integration in $w$ from $l_{3}$ to $i \mathbb{R}$ in 4.15 and identify the space of functions $\left(\mathcal{K}_{31} h\right)(z)$ with $H_{R}(i \mathbb{R})$, the Hardy space on the right half-plane. So, the operator

$$
(\widetilde{\mathcal{K}} h)(z):=\left(\mathcal{K}_{13} \mathcal{K}_{31} h\right)(z)=\frac{\mathcal{C}(z, t)}{\mathcal{A}(z, t)} \int_{l_{1}} \frac{d \widetilde{w}}{2 \pi i} \int_{i \mathbb{R}} \frac{d w}{2 \pi i} \frac{\varphi^{-2}(\widetilde{w})}{z-\widetilde{w}} \frac{\mathcal{B}(\widetilde{w}, t)}{\mathcal{A}(\widetilde{w}, t)} \frac{\varphi_{+}^{2}(w, t)}{\widetilde{w}-w} h(w)
$$

The kernel, $\widetilde{K}(z, w)$ is

$$
\widetilde{K}(z, w)=\frac{\mathcal{C}(z, t)}{\mathcal{A}(z, t)} \varphi_{+}^{2}(w) \int_{l_{1}} \frac{d \widetilde{w}}{2 \pi i} \frac{\varphi^{-2}(\widetilde{w})}{(z-\widetilde{w})(\widetilde{w}-w)} \frac{\mathcal{B}(\widetilde{w}, t)}{\mathcal{A}(\widetilde{w}, t)} .
$$

We can now move $l_{1}$ to $i \mathbb{R}+\epsilon$ from the right without changing the kernel $\widetilde{\mathcal{K}}$

$$
\begin{aligned}
\widetilde{K}(z, w) & =\frac{\mathcal{C}(z, t)}{\mathcal{A}(z, t)} \varphi_{+}^{2}(w) \int_{i \mathbb{R}+\epsilon} \frac{d \widetilde{w}}{2 \pi i} \frac{\varphi_{-}^{-2}(\widetilde{w})}{(z-\widetilde{w})(\widetilde{w}-w)} \frac{\mathcal{B}(\widetilde{w}, t)}{\mathcal{A}(\widetilde{w}, t)} \\
& =\frac{\mathcal{C}(z, t)}{\mathcal{A}(z, t)} \varphi_{+}^{2}(w) \int_{i \mathbb{R}+\epsilon} \frac{d \widetilde{w}}{2 \pi i} \frac{\varphi_{+}^{-2}(\widetilde{w})}{(z-\widetilde{w})(\widetilde{w}-w)} \mathcal{A}(\widetilde{w}, t) \mathcal{B}(\widetilde{w}, t)
\end{aligned}
$$

where in the last identity we use the relation from 4.2$), 4.4): \varphi_{+}(\widetilde{w})=\varphi_{-}(\widetilde{w}) \mathcal{A}(\widetilde{w}, t)$. Therefore we conclude from (4.17) and the above discussion that

$$
\tau_{L U}(t)=\operatorname{det}\left[\mathbb{1}_{L^{2}\left(l_{3}\right)}-\mathcal{K}_{13} \circ \mathcal{K}_{31}\right]=\operatorname{det}\left[\mathbb{1}_{L^{2}(i \mathbb{R})}-\widetilde{\mathcal{K}}\right]
$$

\subsection{Malgrange forms}

In (4.24), we expressed the $\tau$-function on LU as a Fredholm determinant. To relate $\tau_{L U}$ to $\tau_{\Sigma}$ in (3.15), we will first prove that the $\tau$-function corresponding to the RHP 5 , call it $\tau_{L D U}$, is equal to $\tau_{i \mathbb{R}}$ plus non-vanishing explicit factors as in proposition 1, and then show that $\tau_{L U}$ is related to $\tau_{L D U}$ up to explicit terms. We know that the Malgrange form for the RHP on $i \mathbb{R}(3.16)$ is :

$$
\partial_{t} \log \tau_{i \mathbb{R}}=\int_{i \mathbb{R}} \frac{d z}{2 \pi i} \operatorname{Tr}\left[\Theta_{-}^{-1} \Theta_{-}^{\prime} \dot{J} J^{-1}\right] .
$$

Similarly, the Malgrange form of the RHP on LDU 4.2): $Y_{i+1}=Y_{i} F_{i}$ is

$$
\partial_{t} \log \tau_{L D U}=\sum_{i=1}^{3} \int_{l_{i}} \frac{d z}{2 \pi i} \operatorname{Tr}\left[Y_{i}^{-1} Y_{i}^{\prime} \dot{F}_{i} F_{i}^{-1}\right] .
$$

Proposition 3. The Malgrange forms for the RHPs on the contours $i \mathbb{R}$ (RHP这) and on $L D U$ (RHP 5) are related as

$$
\partial_{t} \log \tau_{i \mathbb{R}}=\partial_{t} \log \tau_{L D U}-\int_{i \mathbb{R}} \frac{d z}{2 \pi i}\left(\frac{\dot{\mathcal{B}}}{\mathcal{A}}\right)\left(\mathcal{A C}^{\prime}-\mathcal{A}^{\prime} \mathcal{C}\right) .
$$

the functions $\mathcal{A}, \mathcal{B}, \mathcal{C}$ are defined in (3.4). 
Proof. We begin by substituting (4.1): $J=F_{1} F_{2} F_{3}$ in the term

$$
\begin{aligned}
& \dot{J} J^{-1}=\left(\dot{F}_{1} F_{2} F_{3}+F_{1} \dot{F}_{2} F_{3}+F_{1} F_{2} \dot{F}_{3}\right)\left(F_{3}^{-1} F_{2}^{-1} F_{1}^{-1}\right) \\
& =\left(\dot{F}_{1} F_{1}^{-1}+F_{1} \dot{F}_{2} F_{2}^{-1} F_{1}^{-1}+F_{1} F_{2} \dot{F}_{3} F_{3}^{-1} F_{2}^{-1} F_{1}^{-1}\right) .
\end{aligned}
$$

Substituting in 4.28 in the integrand of 4.25,

$$
\operatorname{Tr}\left[\Theta_{-}^{-1} \Theta_{-}^{\prime} \dot{J} J^{-1}\right]=\operatorname{Tr}\left[\Theta_{-}^{-1} \Theta_{-}^{\prime}\left(\dot{F}_{1} F_{1}^{-1}+F_{1} \dot{F}_{2} F_{2}^{-1} F_{1}^{-1}+F_{1} F_{2} \dot{F}_{3} F_{3}^{-1} F_{2}^{-1} F_{1}^{-1}\right)\right] .
$$

The equivalence (4.3) along with the jump condition 4.2 imply that

$$
\Theta_{-}=Y_{1}, \quad \Theta_{-} F_{1}=Y_{2}, \quad \Theta_{-} F_{1} F_{2}=Y_{3} .
$$

Substituting 4.30 in 4.29,

$$
\begin{gathered}
\operatorname{Tr}\left[\Theta_{-}^{-1} \Theta_{-}^{\prime}\left(\dot{F}_{1} F_{1}^{-1}+F_{1} \dot{F}_{2} F_{2}^{-1} F_{1}^{-1}+F_{1} F_{2} \dot{F}_{3} F_{3}^{-1} F_{2}^{-1} F_{1}^{-1}\right)\right] \\
=\operatorname{Tr}\left[\Theta_{-}^{-1} \Theta_{-}^{\prime} \dot{F}_{1} F_{1}^{-1}+F_{1}^{-1} \Theta_{-}^{-1} \Theta_{-}^{\prime} F_{1} \dot{F}_{2} F_{2}^{-1}+F_{2}^{-1} F_{1}^{-1} \Theta_{-}^{-1} \Theta_{-}^{\prime} F_{1} F_{2} \dot{F}_{3} F_{3}^{-1}\right] \\
=\operatorname{Tr}\left[Y_{1}^{-1} Y_{1}^{\prime} \dot{F}_{1} F_{1}^{-1}+Y_{2}^{-1} Y_{2}^{\prime} \dot{F}_{2} F_{2}^{-1}-F_{1}^{-1} F_{1}^{\prime} \dot{F}_{2} F_{2}^{-1}+Y_{3}^{-1} Y_{3}^{\prime} \dot{F}_{3} F_{3}^{-1}-\left(F_{1} F_{2}\right)^{-1}\left(F_{1} F_{2}\right)^{\prime} \dot{F}_{3} F_{3}^{-1}\right] \\
=\sum_{i=1}^{3} \operatorname{Tr}\left[Y_{i}^{-1} Y_{i}^{\prime} \dot{F}_{i} F_{i}^{-1}\right]-\operatorname{Tr}\left[F_{1}^{-1} F_{1}^{\prime} \dot{F}_{2} F_{2}^{-1}+\left(F_{1} F_{2}\right)^{-1}\left(F_{1} F_{2}\right)^{\prime} \dot{F}_{3} F_{3}^{-1}\right]
\end{gathered}
$$

Therefore,

$$
\begin{aligned}
\int_{i \mathbb{R}} \frac{d z}{2 \pi i} \operatorname{Tr}\left[\Theta_{-}^{-1} \Theta_{-}^{\prime} \dot{J} J^{-1}\right] & =\sum_{i=1}^{3} \int_{l_{i}} \frac{d z}{2 \pi i} \operatorname{Tr}\left[Y_{i}^{-1} Y_{i}^{\prime} \dot{F}_{i} F_{i}^{-1}\right]-\int_{i \mathbb{R}} \frac{d z}{2 \pi i} \operatorname{Tr}\left[F_{1}^{-1} F_{1}^{\prime} \dot{F}_{2} F_{2}^{-1}\right] \\
& -\int_{i \mathbb{R}} \frac{d z}{2 \pi i} \operatorname{Tr}\left[\left(F_{1} F_{2}\right)^{-1}\left(F_{1} F_{2}\right)^{\prime} \dot{F}_{3} F_{3}^{-1}\right]
\end{aligned}
$$

Let us analyze the explicit terms.

- Since $F_{1}$ is upper triangular and $F_{2}$ is diagonal as defined in 4.1,

$$
\operatorname{Tr}\left[F_{1}^{-1} F_{1}^{\prime} \dot{F}_{2} F_{2}^{-1}\right]=0 \text {. }
$$

- Substituting $F_{1,2,3}$ in the last term in 4.32 ,

$$
\operatorname{Tr}\left[\left(F_{1} F_{2}\right)^{-1}\left(F_{1} F_{2}\right)^{\prime} \dot{F}_{3} F_{3}^{-1}\right]=\left(\frac{\dot{\mathcal{B}}}{\mathcal{A}}\right)\left(\mathcal{A C}^{\prime}-\mathcal{A}^{\prime} \mathcal{C}\right)
$$

where $\mathcal{A}, \mathcal{B}, \mathcal{C}$ are explicit in terms of parabolic cylinder functions 3.4.

Therefore,

$$
\partial_{t} \log \tau_{i \mathbb{R}}=\partial_{t} \log \tau_{L D U}-\int_{i \mathbb{R}} \frac{d z}{2 \pi i}\left(\frac{\dot{\mathcal{B}}}{\mathcal{A}}\right)\left(\mathcal{A C}^{\prime}-\mathcal{A}^{\prime} \mathcal{C}\right)
$$


Recall from proposition 3 , the Malgrange form of the RHP on LDU 4.26):

$$
\partial_{t} \log \tau_{L D U}=\sum_{i=1}^{3} \int_{l_{i}} \frac{d z}{2 \pi i} \operatorname{Tr}\left[Y_{i}^{-1} Y_{i}^{\prime} \dot{F}_{i} F_{i}^{-1}\right] .
$$

For the RHP on LU (RHP 6 with the jump condition 44.6): $\tilde{Y}_{i+1}=\widetilde{Y}_{i} \widetilde{F}_{i}$ where $i=1,3$, the Malgrange form reads

$$
\partial_{t} \log \tau_{L U}=\sum_{i=1,3} \int_{l_{i}} \frac{d z}{2 \pi i} \operatorname{Tr}\left[\widetilde{Y}_{i}^{-1} \widetilde{Y}_{i}^{\prime} \dot{\widetilde{F}}_{i} \widetilde{F}_{i}^{-1}\right] .
$$

Proposition 4. The Malgrange forms of the RHPs on contours LDU (RHP 5) and LU $($ RHP $\sqrt{6})$ are related as

$$
\partial_{t} \log \tau_{L D U}=\partial_{t} \log \tau_{L U}+2 \int_{i \mathbb{R}} \frac{d z}{2 \pi i} \frac{\dot{\mathcal{A}}(z, t)}{\mathcal{A}(z, t)} \int_{i \mathbb{R}_{-}} \frac{d w}{2 \pi i} \frac{\mathcal{A}^{\prime}(w, t)}{\mathcal{A}(w, t)(z-w)}
$$

Proof. We will first simplify the integrals on $l_{1}$ and $l_{3}$ in 4.36$)$. Given that 4.5$): Y_{i}=\widetilde{Y}_{i} \varphi^{\sigma_{3}}$ and $4.10: F_{i}=\varphi^{-\sigma_{3}} \widetilde{F}_{i} \varphi^{\sigma_{3}}$

$$
\begin{gathered}
\sum_{i=1,3} \int_{l_{i}} \frac{d z}{2 \pi i} \operatorname{Tr}\left[Y_{i}^{-1} Y_{i}^{\prime} \dot{F}_{i} F_{i}^{-1}\right] \\
=\sum_{i=1,3} \int_{l_{i}} \frac{d z}{2 \pi i} \operatorname{Tr}\left[\left(\widetilde{Y}_{i} \varphi^{\sigma_{3}}\right)^{-1}\left(\widetilde{Y}_{i-} \varphi^{\sigma_{3}}\right)^{\prime} \partial_{t}\left(\varphi^{-\sigma_{3}} \widetilde{F}_{i} \varphi^{\sigma_{3}}\right)\left(\varphi^{-\sigma_{3}} \widetilde{F}_{i}^{-1} \varphi^{\sigma_{3}}\right)\right] \\
=\sum_{i=1,3} \int_{l_{i}} \frac{d z}{2 \pi i} \operatorname{Tr}\left[\left(\widetilde{Y}_{i} \varphi^{\sigma_{3}}\right)^{-1}\left(\widetilde{Y}_{i} \varphi^{\sigma_{3}}\right)^{\prime} \partial_{t}\left(\varphi^{-\sigma_{3}} \widetilde{F}_{i} \varphi^{\sigma_{3}}\right)\left(\varphi^{-\sigma_{3}} \widetilde{F}_{i}^{-1} \varphi^{\sigma_{3}}\right)\right] \\
=\sum_{i=1,3} \int_{l_{i}} \frac{d z}{2 \pi i} \operatorname{Tr}\left[\left(\widetilde{Y}_{i}^{-1} \widetilde{Y}_{i}^{\prime}+\sigma_{3} \varphi^{\prime} \varphi^{-1}\right)\left(-\sigma_{3} \dot{\varphi} \varphi^{-1}+\dot{\widetilde{F}}_{i} \widetilde{F}_{i}^{-1}+\widetilde{F}_{i} \sigma_{3} \dot{\varphi} \varphi^{-1} \widetilde{F}_{i}^{-1}\right)\right] \\
=\sum_{i=1,3} \int_{l_{1}} \frac{d z}{2 \pi i} \operatorname{Tr}\left[\widetilde{Y}_{i-}^{-1} \widetilde{Y}_{i-}^{\prime} \dot{\widetilde{F}}_{i} \widetilde{F}_{i}^{-1}\right]+\sum_{i=1,3} \int_{l_{i}} \frac{d z}{2 \pi i} \operatorname{Tr}\left[\left(\widetilde{Y}_{i}^{-1} \widetilde{Y}_{i}^{\prime}\right)\left(-\sigma_{3} \dot{\varphi} \varphi^{-1}+\widetilde{F}_{i} \sigma_{3} \dot{\varphi} \varphi^{-1} \widetilde{F}_{i}^{-1}\right)\right] \\
+\sum_{i=1,3} \int_{l_{i}} \frac{d z}{2 \pi i} \operatorname{Tr}\left[\sigma_{3} \varphi^{\prime} \varphi^{-1}\left(-\sigma_{3} \dot{\varphi} \varphi^{-1}+\dot{\widetilde{F}}_{i} \widetilde{F}_{i}^{-1}+\widetilde{F}_{i} \sigma_{3} \dot{\varphi} \varphi^{-1} \widetilde{F}_{i}^{-1}\right)\right] .
\end{gathered}
$$

In 4.39, $\widetilde{F}_{i}$ are either lower or upper triangular 4.10. Therefore,

$$
\int_{l_{1} \cup l_{3}} \frac{d z}{2 \pi i} \operatorname{Tr}\left[\sigma_{3} \varphi^{\prime} \varphi^{-1}\left(-\sigma_{3} \dot{\varphi} \varphi^{-1}+\dot{\widetilde{F}}_{i} \widetilde{F}_{i}^{-1}+\widetilde{F}_{i} \sigma_{3} \dot{\varphi} \varphi^{-1} \widetilde{F}_{i}^{-1}\right)\right]=0
$$

Therefore, given 4.37, 4.39 reads

$$
\begin{array}{r}
\sum_{i=1,3} \int_{l_{i}} \frac{d z}{2 \pi i} \operatorname{Tr}\left[Y_{i}^{-1} Y_{i}^{\prime} \dot{F}_{i} F_{i}^{-1}\right]=\sum_{i=1,3} \int_{l_{i}} \frac{d z}{2 \pi i} \operatorname{Tr}\left[\widetilde{Y}_{i}^{-1} \widetilde{Y}_{i}^{\prime} \dot{\widetilde{F}}_{i} \widetilde{F}_{i}^{-1}\right] \\
+\sum_{i=1,3} \int_{l_{i}} \frac{d z}{2 \pi i} \operatorname{Tr}\left[\left(\widetilde{Y}_{i}^{-1} \widetilde{Y}_{i}^{\prime}\right)\left(-\sigma_{3} \dot{\varphi} \varphi^{-1}+\widetilde{F}_{i} \sigma_{3} \dot{\varphi} \varphi^{-1} \widetilde{F}_{i}^{-1}\right)\right] \\
=\partial_{t} \log \tau_{L U}+\sum_{i=1,3} \int_{l_{i}} \frac{d z}{2 \pi i} \operatorname{Tr}\left[\left(\widetilde{Y}_{i}^{-1} \widetilde{Y}_{i}^{\prime}\right)\left(-\sigma_{3} \dot{\varphi} \varphi^{-1}+\widetilde{F}_{i} \sigma_{3} \dot{\varphi} \varphi^{-1} \widetilde{F}_{i}^{-1}\right)\right] .
\end{array}
$$


Recalling 4.6): $\widetilde{Y}_{i+1}=\widetilde{Y}_{i} \widetilde{F}_{i}$, the second term in 4.41) can be further simplified

$$
\begin{gathered}
\sum_{i=1,3} \int_{l_{i}} \frac{d z}{2 \pi i} \operatorname{Tr}\left[\left(\widetilde{Y}_{i}^{-1} \widetilde{Y}_{i}^{\prime}\right)\left(-\sigma_{3} \dot{\varphi} \varphi^{-1}+\widetilde{F}_{i} \sigma_{3} \dot{\varphi} \varphi^{-1} \widetilde{F}_{i}^{-1}\right)\right] \\
=\sum_{i=1,3} \int_{l_{i}} \frac{d z}{2 \pi i} \operatorname{Tr}\left[-\widetilde{Y}_{i}^{-1} \widetilde{Y}_{i}^{\prime} \sigma_{3} \dot{\varphi} \varphi^{-1}+\widetilde{F}_{i}^{-1} \widetilde{Y}_{i}^{-1} \widetilde{Y}_{i}^{\prime} \widetilde{F}_{i} \sigma_{3} \dot{\varphi} \varphi^{-1}\right] \\
=\sum_{i=1,3} \int_{l_{i}} \frac{d z}{2 \pi i} \operatorname{Tr}\left[-\widetilde{Y}_{i}^{-1} \widetilde{Y}_{i}^{\prime} \sigma_{3} \dot{\varphi} \varphi^{-1}+\widetilde{Y}_{i+1}^{-1} \widetilde{Y}_{i+1}^{\prime} \sigma_{3} \dot{\varphi} \varphi^{-1}-\widetilde{F}_{i}^{-1} \widetilde{F}_{i}^{\prime} \sigma_{3} \dot{\varphi} \varphi^{-1}\right] \\
=\sum_{i=1,3} \int_{l_{i}} \frac{d z}{2 \pi i} \operatorname{Tr}\left[\Delta\left(\widetilde{Y}_{i}^{-1} \widetilde{Y}_{i}^{\prime}\right) \sigma_{3} \dot{\varphi} \varphi^{-1}-\widetilde{F}_{i}^{-1} \widetilde{F}_{i}^{\prime} \sigma_{3} \dot{\varphi} \varphi^{-1}\right] \\
=\sum_{i=1,3} \int_{l_{i}} \frac{d z}{2 \pi i} \operatorname{Tr}\left[\Delta\left(\widetilde{Y}_{i}^{-1} \widetilde{Y}_{i}^{\prime}\right) \sigma_{3} \dot{\varphi} \varphi^{-1}\right]
\end{gathered}
$$

where now $\Delta\left(\widetilde{Y}_{i}^{-1} \widetilde{Y}_{i}^{\prime}\right)=\widetilde{Y}_{i+1}^{-1} \widetilde{Y}_{i+1}^{\prime}-\widetilde{Y}_{i}^{-1} \widetilde{Y}_{i}^{\prime}$. The last line is obtained using the fact that $\operatorname{Tr}\left[\widetilde{F}_{i}^{-1} \widetilde{F}_{i}^{\prime} \sigma_{3} \dot{\varphi} \varphi^{-1}\right]=0$ since $\widetilde{F}_{i}$ is either lower or upper triangular and $\varphi$ is scalar.

The final expression in 4.42 can be further simplified by noting that the function $\varphi$ has no jumps on $l_{1}$ and $l_{3}$. Beginning with the integral on $l_{1}$,

$$
\begin{gathered}
\int_{l_{1}} \frac{d z}{2 \pi i} \operatorname{Tr}\left[\Delta\left(\tilde{Y}_{1}^{-1} \tilde{Y}_{1}^{\prime}\right) \sigma_{3} \dot{\varphi} \varphi^{-1}\right]=\int_{l_{1}} \frac{d z}{2 \pi i} \operatorname{Tr}\left[\left(\widetilde{Y}_{2}^{-1} \tilde{Y}_{2}^{\prime}-\widetilde{Y}_{1}^{-1} \tilde{Y}_{1}^{\prime}\right) \sigma_{3} \dot{\varphi} \varphi^{-1}\right] \\
=\int_{l_{1}} \frac{d z}{2 \pi i} \operatorname{Tr}\left[\widetilde{Y}_{2}^{-1} \widetilde{Y}_{2}^{\prime} \sigma_{3} \dot{\varphi} \varphi^{-1}\right] .
\end{gathered}
$$

To obtain the last line, we notice from fig. 8 that $\int_{l_{1}} \frac{d z}{2 \pi i} \operatorname{Tr}\left[\widetilde{Y}_{1}^{-1} \widetilde{Y}_{1}^{\prime} \sigma_{3} \dot{\varphi} \varphi^{-1}\right]=0$ by closing the contour on the right. A similar computation follows for the integral on $l_{3}$ in 4.42

$$
\begin{gathered}
\int_{l_{3}} \frac{d z}{2 \pi i} \operatorname{Tr}\left[\Delta\left(\widetilde{Y}_{3}^{-1} \widetilde{Y}_{3}^{\prime}\right) \sigma_{3} \dot{\varphi} \varphi^{-1}\right]=\int_{l_{3}} \frac{d z}{2 \pi i} \operatorname{Tr}\left[\left(\widetilde{Y}_{4}^{-1} \widetilde{Y}_{4}^{\prime}-\widetilde{Y}_{3}^{-1} \widetilde{Y}_{3}^{\prime}\right) \sigma_{3} \dot{\varphi} \varphi^{-1}\right] \\
=-\int_{l_{3}} \frac{d z}{2 \pi i} \operatorname{Tr}\left[\widetilde{Y}_{3}^{-1} \widetilde{Y}_{3}^{\prime} \sigma_{3} \dot{\varphi} \varphi^{-1}\right] .
\end{gathered}
$$

To obtain the last line, we note that $\int_{l_{3}} \frac{d z}{2 \pi i} \operatorname{Tr}\left[\widetilde{Y}_{4}^{-1} \widetilde{Y}_{4}^{\prime} \sigma_{3} \dot{\varphi} \varphi^{-1}\right]=0$ by closing the contour on the left (see fig. 8).

Gathering the terms 4.43), 4.44), and using 4.6): $\widetilde{Y}_{2}=\widetilde{Y}_{3}$, 4.42 reads

$$
\begin{gathered}
\sum_{i=1,3} \int_{l_{i}} \frac{d z}{2 \pi i} \operatorname{Tr}\left[\Delta\left(\widetilde{Y}_{i}^{-1} \widetilde{Y}_{i}^{\prime}\right) \sigma_{3} \dot{\varphi} \varphi^{-1}\right]=\int_{l_{1}} \frac{d z}{2 \pi i} \operatorname{Tr}\left[\widetilde{Y}_{2}^{-1} \widetilde{Y}_{2}^{\prime} \sigma_{3} \dot{\varphi} \varphi^{-1}\right]-\int_{l_{3}} \frac{d z}{2 \pi i} \operatorname{Tr}\left[\widetilde{Y}_{3}^{-1} \widetilde{Y}_{3}^{\prime} \sigma_{3} \dot{\varphi} \varphi^{-1}\right] \\
=-\int_{l_{2}} \frac{d z}{2 \pi i} \operatorname{Tr}\left[\widetilde{Y}_{2}^{-1} \widetilde{Y}_{2}^{\prime} \sigma_{3} \dot{\varphi} \varphi^{-1}\right]+\int_{l_{2}} \frac{d z}{2 \pi i} \operatorname{Tr}\left[\widetilde{Y}_{3}^{-1} \widetilde{Y}_{3}^{\prime} \sigma_{3} \dot{\varphi} \varphi^{-1}\right]=0
\end{gathered}
$$

Substituting 4.45 in 4.41,

$$
\sum_{i=1,3} \int_{l_{i}} \frac{d z}{2 \pi i} \operatorname{Tr}\left[Y_{i}^{-1} Y_{i}^{\prime} \dot{F}_{i} F_{i}^{-1}\right]=\sum_{i=1,3} \int_{l_{i}} \frac{d z}{2 \pi i} \operatorname{Tr}\left[\widetilde{Y}_{i}^{-1} \widetilde{Y}_{i}^{\prime} \dot{\widetilde{F}}_{i} \widetilde{F}_{i}^{-1}\right]=\partial_{t} \log \tau_{L U}
$$


We now compute the integral on $l_{2}$ in 4.39

$$
\begin{gathered}
\int_{l_{2}} \frac{d z}{2 \pi i} \operatorname{Tr}\left[Y_{2}^{-1} Y_{2}^{\prime} \dot{F}_{2} F_{2}^{-1}\right]=\int_{l_{2}} \frac{d z}{2 \pi i} \operatorname{Tr}\left[\left(\widetilde{Y}_{2} \varphi_{-}^{\sigma_{3}}\right)^{-1}\left(\widetilde{Y}_{2}^{\prime} \varphi_{-}^{\sigma_{3}}+\widetilde{Y}_{2}\left(\varphi_{-}^{\sigma_{3}}\right)^{\prime}\right) \dot{F}_{2} F_{2}^{-1}\right] \\
=\int_{l_{2}} \frac{d z}{2 \pi i} \operatorname{Tr}\left[\varphi_{-}^{-\sigma_{3}} \widetilde{Y}_{2}^{-1}\left(\widetilde{Y}_{2}^{\prime} \varphi_{-}^{\sigma_{3}}+\widetilde{Y}_{2}\left(\varphi_{-}^{\sigma_{3}}\right)^{\prime}\right) \dot{F}_{2} F_{2}^{-1}\right] \\
=\int_{l_{2}} \frac{d z}{2 \pi i} \operatorname{Tr}\left[\widetilde{Y}_{2}^{-1} \widetilde{Y}_{2}^{\prime} \dot{F}_{2} F_{2}^{-1}+\sigma_{3} \varphi_{-}^{-1} \varphi_{-}^{\prime} \dot{F}_{2} F_{2}^{-1}\right]
\end{gathered}
$$

Since $\widetilde{Y}_{2}$ does not jump on $l_{2}$, Liouville theorem implies that

$$
\operatorname{Tr}\left[\widetilde{Y}_{2}^{-1} \widetilde{Y}_{2}^{\prime} \dot{F}_{2} F_{2}^{-1}\right]=0
$$

The term

$$
\operatorname{Tr}\left[\sigma_{3} \varphi_{-}^{-1} \varphi_{-}^{\prime} \dot{F}_{2} F_{2}^{-1}\right]
$$

in 4.47) is an explicit function of $\mathcal{A}(z, w)$ in 3.4. From 4.1),

$$
F_{2}=\mathcal{A}^{\sigma_{3}} \Rightarrow \dot{F}_{2} F_{2}^{-1}=\frac{\dot{\mathcal{A}}}{\mathcal{A}} \sigma_{3}
$$

The function $\varphi_{-}$is the boundary value of $\varphi$ defined in 4.4

$$
\varphi_{-}=\exp \left[\int_{i \mathbb{R}-\epsilon} \frac{d w}{2 \pi i} \frac{\log \mathcal{A}(w, t)}{z-w}\right] \Rightarrow \varphi_{-}^{-1} \varphi_{-}^{\prime}=\int_{i \mathbb{R}-\epsilon} \frac{d w}{2 \pi i} \frac{\mathcal{A}^{\prime}(w, t)}{\mathcal{A}(w, t)(z-w)} .
$$

The expression 4.47) simplifies as follows due to 4.50, 4.51

$$
\begin{gathered}
\int_{l_{2}} \frac{d z}{2 \pi i} \operatorname{Tr}\left[Y_{2}^{-1} Y_{2}^{\prime} \dot{F}_{2} F_{2}^{-1}\right]=\int_{l_{2}} \frac{d z}{2 \pi i} \operatorname{Tr}\left[\sigma_{3} \varphi_{-}^{-1} \varphi_{-}^{\prime} \dot{F}_{2} F_{2}^{-1}\right] \\
=\int_{i \mathbb{R}} \frac{d z}{2 \pi i}\left(2 \frac{\dot{\mathcal{A}}(z, t)}{\mathcal{A}(z, t)}\right) \int_{i \mathbb{R}-\epsilon} \frac{d w}{2 \pi i} \frac{\mathcal{A}^{\prime}(w, t)}{\mathcal{A}(w, t)(z-w)}
\end{gathered}
$$

Substituting 4.52 and 4.46, in 4.36

$$
\begin{gathered}
\partial_{t} \log \tau_{L D U}=\sum_{i=1}^{3} \int_{l_{i}} \frac{d z}{2 \pi i} \operatorname{Tr}\left[Y_{i}^{-1} Y_{i}^{\prime} \dot{F}_{i} F_{i}^{-1}\right] \\
=\sum_{i=1,3} \int_{l_{i}} \frac{d z}{2 \pi i} \operatorname{Tr}\left[Y_{i}^{-1} Y_{i}^{\prime} \dot{F}_{i} F_{i}^{-1}\right]+\int_{i \mathbb{R}} \frac{d z}{2 \pi i} \operatorname{Tr}\left[\sigma_{3} \varphi_{-}^{-1} \varphi_{-}^{\prime} \dot{F}_{2} F_{2}^{-1}\right] \\
=\partial_{t} \log \tau_{L U}+2 \int_{i \mathbb{R}} \frac{d z}{2 \pi i} \frac{\dot{\mathcal{A}}(z, t)}{\mathcal{A}(z, t)} \int_{i \mathbb{R}_{-}} \frac{d w}{2 \pi i} \frac{\mathcal{A}^{\prime}(w, t)}{\mathcal{A}(w, t)(z-w)}
\end{gathered}
$$




\section{Proof of theorem 1}

Proof. The propositions 1, 3, 4 imply that the $\tau$-functions $\tau_{\Sigma}$ and $\tau_{L U}$ are related through explicit factors, and the proposition 2 expresses $\tau_{L U}$ as a Fredholm determinant. Therefore, the $\tau$-function of Painleve II equation defined in 2.14

$$
\begin{gathered}
\partial_{t} \log \tau_{P I I} \equiv \partial_{t} \log \tau_{\Sigma} \stackrel{\sqrt[3.41]{-}}{=} \partial_{t} \log \tau_{i \mathbb{R}}-\int_{i \mathbb{R}} \frac{d z}{2 \pi i} \widetilde{\mathcal{F}}(z, t ; \nu, h)-\left[\frac{4 i \nu}{3}+\frac{2 \nu^{2}}{t}\right] \\
\stackrel{4.35]}{-} \partial_{t} \log \tau_{L D U}-\int_{i \mathbb{R}} \frac{d z}{2 \pi i}\left\{\left(\frac{\dot{\mathcal{B}}}{\mathcal{A}}\right)\left(\mathcal{A} \mathcal{C}^{\prime}-\mathcal{A}^{\prime} \mathcal{C}\right)+\widetilde{\mathcal{F}}(\zeta, \xi, t ; \nu, h)\right\}-\left[\frac{4 i \nu}{3}+\frac{2 \nu^{2}}{t}\right] \\
\stackrel{[4.53)}{-} \partial_{t} \log \tau_{L U}+\int_{i \mathbb{R}} \frac{d z}{2 \pi i}\left\{\frac{2 \dot{\mathcal{A}}(z, t)}{\mathcal{A}(z, t)}\left(\int_{i \mathbb{R}-\epsilon} \frac{d w}{2 \pi i} \frac{\mathcal{A}^{\prime}(w, t)}{\mathcal{A}(w, t)(z-w)}\right)-\left(\frac{\dot{\mathcal{B}}}{\mathcal{A}}\right)\left(\mathcal{A} \mathcal{C}^{\prime}-\mathcal{A}^{\prime} \mathcal{C}\right)-\widetilde{\mathcal{F}}(z, t ; \nu, h)\right\} \\
-\left[\frac{4 i \nu}{3}+\frac{2 \nu^{2}}{t}\right] \\
+\int_{i \mathbb{R}} \frac{d z}{2 \pi i}\left\{\frac{2 \dot{\mathcal{A}}(z, t)}{\mathcal{A}(z, t)}\left(\int_{i \mathbb{R}-\epsilon} \frac{d w}{2 \pi i} \frac{\mathcal{A}^{\prime}(w, t)}{\mathcal{A}(w, t)(z-w)}\right)-\left(\frac{\dot{\mathcal{B}}}{\mathcal{A}}\right)\left(\mathcal{A C} \mathcal{C}^{\prime}-\mathcal{A}^{\prime} \mathcal{C}\right)-\widetilde{\mathcal{F}}(z, t ; \nu, h)\right\} .
\end{gathered}
$$

In (5.1), the functions $\mathcal{A}, \mathcal{B}, \mathcal{C}$ defined in (3.4) are explicit in terms of parabolic cylinder functions, $\widetilde{\mathcal{F}}$ is defined in 3.40 , and the term

$$
\int_{i \mathbb{R}} \frac{d z}{2 \pi i}\left\{\frac{2 \dot{\mathcal{A}}(z, t)}{\mathcal{A}(z, t)}\left(\int_{i \mathbb{R}-\epsilon} \frac{d w}{2 \pi i} \frac{\mathcal{A}^{\prime}(w, t)}{\mathcal{A}(w, t)(z-w)}\right)-\left(\frac{\dot{\mathcal{B}}}{\mathcal{A}}\right)\left(\mathcal{A C}^{\prime}-\mathcal{A}^{\prime} \mathcal{C}\right)-\widetilde{\mathcal{F}}(z, t ; \nu, h)\right\}
$$

depends only on $h, \nu$ and $t$. We then define

$$
\mathcal{F}(t, \nu, h):=\int_{i \mathbb{R}} \frac{d z}{2 \pi i}\left\{\frac{2 \dot{\mathcal{A}}(z, t)}{\mathcal{A}(z, t)}\left(\int_{i \mathbb{R}-\epsilon} \frac{d w}{2 \pi i} \frac{\mathcal{A}^{\prime}(w, t)}{\mathcal{A}(w, t)(z-w)}\right)-\left(\frac{\dot{\mathcal{B}}}{\mathcal{A}}\right)\left(\mathcal{A} \mathcal{C}^{\prime}-\mathcal{A}^{\prime} \mathcal{C}\right)-\widetilde{\mathcal{F}}(z, t ; \nu, h)\right\} .
$$

In terms of $\mathcal{F}(t, \nu, h)$, 5.1 reads

$$
\partial_{t} \log \tau_{P I I}=\partial_{t} \log \operatorname{det}\left[\mathbb{1}_{L^{2}(i \mathbb{R})}-\widetilde{\mathcal{K}}\right]+F(t, \nu, h)-\left[\frac{4 i \nu}{3}+\frac{2 \nu^{2}}{t}\right] .
$$

Therefore, the $\tau$-function of Painlevé II can be expressed as a Fredholm determinant of an integrable operator up to explicit factors. Furthermore, solving the RHP 6 is equivalent to solving the RHP 4, which in turn is tantamount to solving the RHP2. Therefore, the zeros of $\tau_{P I I}$ (solvability condition of 2 ) are completely determined by the zeros of the Fredholm determinant 4.24. 


\section{References}

[1] M Bertola. "On the location of poles for the Ablowitz-Segur family of solutions to the second Painlevé equation". In: Nonlinearity 25.4 (2012), p. 1179. DOI: 10.1088/ 0951-7715/25/4/1179.

[2] M Bertola. "The Malgrange Form and Fredholm Determinants". In: Symmetry, Integrability and Geometry: Methods and Applications 13.0 (2017), pp. 46-12. DOI: 10.3842/SIGMA.2017.046.

[3] M Bertola, JGE Rebelo, and T Grava. "Painlevé IV Critical Asymptotics for Orthogonal Polynomials in the Complex Plane." In: SIGMA 14 (2018), p. 091. DOI: 10.3842/SIGMA.2018.091.

[4] A Bolibruch, A Its, and A Kapaev. "On the Riemann-Hilbert-Birkhoff inverse monodromy problem and the Painlevé equations". In: St. Petersburg Mathematical Journal 16.1 (2005), pp. 105-142. DOI: 10.1090/S1061-0022-04-00845-3.

[5] F Bornemann. "On the numerical evaluation of Fredholm determinants". In: Mathematics of Computation 79.270 (2010), pp. 871-915. DOI: 10.1090/S0025-5718-09$02280-7$.

[6] A Borodin and P Deift. "Fredholm determinants, Jimbo-Miwa-Ueno $\tau$-functions, and representation theory". In: Communications on Pure and Applied Mathematics 55.9 (2002), pp. 1160-1230. DOI: 10.1002/cpa.10042.

[7] A Borodin and G Olshanski. "Distributions on Partitions, Point Processes, and the Hypergeometric Kernel". In: Communications in Mathematical Physics 211.2 (2000), pp. 335-358. DOI: $10.1007 / \mathrm{s} 002200050815$.

[8] T Bothner. "Transition asymptotics for the Painlevé II transcendent". In: Duke Mathematical Journal 166.2 (2017), pp. 205-324. DOI: 10.1215/00127094-3714650.

[9] T Bothner and R Buckingham. "Large deformations of the Tracy-Widom distribution I: non-oscillatory asymptotics". In: Communications in Mathematical Physics 359.1 (2018), pp. 223-263. DOI: 10.1007/s00220-017-3006-7.

[10] T Bothner and A Its. Asymptotics of a Fredholm determinant involving the second Painlevé transcendent. arXiv: 1209.5415.

[11] T Bothner and A Its. "The nonlinear steepest descent approach to the singular asymptotics of the second Painlevé transcendent". In: Physica D: Nonlinear Phenomena 241.23-24 (2012), pp. 2204-2225. DOI: 10.1016/j . physd.2012.02.014.

[12] M Cafasso, P Gavrylenko, and O Lisovyy. "Tau functions as Widom constants". In: Communications in Mathematical Physics 365.2 (2019), pp. 741-772. DOI: 10.1007/ s00220-018-3230-9.

[13] PA Clarkson. "Painlevé equations - nonlinear special functions". In: Orthogonal polynomials and special functions. Springer, 2006, pp. 331-411.

[14] P Deift. "Integrable operators". English (US). In: American Mathematical Society Translations 189.2 (1999), pp. 69-84.

[15] P Deift. "Riemann-Hilbert problems". In: Random Matrices. Ed. by A Borodin, I Corwin, and A Guionnet. American Mathematical Soc., 2019. Chap. 1, pp. 1-40.

[16] PA Deift and X Zhou. "Asymptotics for the Painlevé II equation". In: Communications on Pure and Applied Mathematics 48.3 (1995), pp. 277-337. DOI: 10.1002/ cpa.3160480304. 
[17] H Desiraju. "The $\tau$-function of the Ablowitz-Segur family of solutions to Painlevé II as a Widom constant". In: Journal of Mathematical Physics 60.11 (2019), p. 113505. DOI: $10.1063 / 1.5120357$.

[18] H Flaschka and A Newell. "Monodromy-and spectrum-preserving deformations I". In: Communications in Mathematical Physics 76.1 (1980), pp. 65-116. DOI: 10 . 1007/BF01197110.

[19] AS Fokas et al. Painlevé transcendents: the Riemann-Hilbert approach. 128. American Mathematical Soc., 2006.

[20] B Fornberg and JAC Weideman. "A computational exploration of the second Painlevé equation". In: Foundations of Computational Mathematics 14.5 (2014), pp. 985-1016. DOI: $10.1007 / \mathrm{s} 10208-013-9156-\mathrm{x}$.

[21] PJ Forrester and NS Witte. "Application of the $\tau$-function Theory of Painlevé Equations to Random Matrices: PIV, PII and the GUE". In: Communications in Mathematical Physics 219.2 (2001), pp. 357-398. DOI: 10.1007/s002200100422.

[22] PJ Forrester and NS Witte. "Painlevé II in random matrix theory and related fields". In: Constructive Approximation 41.3 (2015), pp. 589-613. DOI: $10.1007 /$ s00365014-9243-5.

[23] P Gavrylenko and O Lisovyy. "Fredholm determinant and Nekrasov sum representations of isomonodromic tau functions". In: Communications in Mathematical Physics 363.1 (2018), pp. 1-58. DOI: 10.1007/s00220-018-3224-7.

[24] IS Gradshteyn and IM Ryzhik. Table of integrals, series, and products. Academic press, 2014.

[25] P Howes and N Joshi. "Global asymptotics of the second Painlevé equation in Okamoto's space". In: Constructive Approximation 39.1 (2014), pp. 11-41. DOI: 10.1007/s00365-013-9222-2.

[26] AR Its and AA Kapaev. "The nonlinear steepest descent approach to the asymptotics of the second Painleve transcendent in the complex domain". In: MathPhys Odyssey 2001. Springer, 2002, pp. 273-311.

[27] AR Its, O Lisovyy, and A Prokhorov. "Monodromy dependence and connection formulae for isomonodromic tau functions". In: Duke Mathematical Journal 167.7 (2018), pp. 1347-1432. DOI: 10.1215/00127094-2017-0055.

[28] AR Its et al. "Differential equations for quantum correlation functions". In: International Journal of Modern Physics B 4.05 (1990), pp. 1003-1037. DOI: 10.1142/ S0217979290000504.

[29] M Jimbo, T Miwa, and K Ueno. "Monodromy preserving deformation of linear ordinary differential equations with rational coefficients: I. General theory and $\tau$ function". In: 1981. DOI: 10.1016/0167-2789(81)90013-0.

[30] N Joshi, K Kajiwara, and M Mazzocco. "Generating function associated with the determinant formula for the solutions of the Painleve II equation". In: Asterisque 297 (2004), pp. 67-78. URL: https://arxiv.org/abs/nlin/0406035.

[31] K Kajiwara and T Masuda. "A generalization of determinant formulae for the solutions of Painlevé II and XXXIV equations". In: Journal of Physics A: Mathematical and General 32.20 (1999), p. 3763. DOI: 10.1088/0305-4470/32/20/309.

[32] A Kapaev. "Global asymptotics of the second Painlevé transcendent". In: Physics Letters A 167.4 (1992), pp. 356-362. DOI: 10.1016/0375-9601(92)90271-M. 
[33] B Malgrange. "Déformations isomonodromiques des singularités réguliéres". In: Les rencontres physiciens-mathématiciens de Strasbourg-RCP25 31 (1983), pp. 1-26. URL: http://www.numdam.org/item/CIF_1982__17__A2_0/.

[34] V Yu Novokshenov. "Padé approximations for Painlevé I and II transcendents". In: Theoretical and Mathematical Physics 159.3 (2009), pp. 853-862. DOI: 10.1007/ s11232-009-0073-8.

[35] K Okamoto. "Studies on the Painlevé equations. III: Second and fourth Painlevé equations, PII and PIV". In: Mathematische Annalen 275.2 (1986), pp. 221-255.

[36] J Palmer. "Determinants of Cauchy-Riemann operators as $\tau$-functions". In: Acta Applicandae Mathematica 18.3 (1990), pp. 199-223. DOI: 10.1007/BF00049126.

[37] V Retakh and V Rubtsov. "Noncommutative Toda chains, Hankel quasideterminants and the Painlevé II equation". In: Journal of Physics A: Mathematical and Theoretical 43.50 (Nov. 2010), p. 505204. DOI: 10.1088/1751-8113/43/50/505204.

[38] H Segur and MJ Ablowitz. "Asymptotic solutions of nonlinear evolution equations and a Painlevé transcedent". In: Physica D: Nonlinear Phenomena 3.1-2 (1981), pp. 165-184. DOI: 10.1016/0167-2789(81)90124-X.

[39] CA Tracy and H Widom. "Airy kernel and Painlevé II". In: vol. 31. 2002, pp. 85-98. URL: https://arxiv.org/abs/solv-int/9901004v2.

[40] CA Tracy and H Widom. "Fredholm determinants, differential equations and matrix models". In: Communications in mathematical physics 163.1 (1994), pp. 33-72. DOI: 10.1007/BF02101734.

[41] CA Tracy and H Widom. "Level spacing distributions and the Bessel kernel". In: Communications in mathematical physics 161.2 (1994), pp. 289-309. DOI: 10.1007/ BF02099779.

[42] CA Tracy and H Widom. "Level-spacing distributions and the Airy kernel". In: Communications in Mathematical Physics 159.1 (1994), pp. 151-174. DOI: 10.1007/ BF02100489. 